Pacific Journal of Mathematics

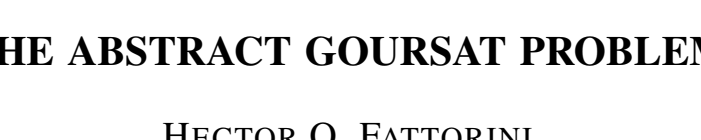




\section{THE ABSTRACT GOURSAT PROBLEM}

\section{H. O. FATTORINI}

Let $D^{\alpha}$ be a differential monomial in $n$ variables. We try to identify in this paper those closed, densely defined linear operators $A$ (in a fairly general class of locally convex spaces) such that the equation $\left(D^{\alpha}-A\right) S=\delta \otimes I$ has a solution; here $S$ is an operator-valued distribution in $n$ variables with support in the cone of nonnegative coordinates. The results are applied to the study of the equation $\left(D^{\alpha}-A\right) U=T, T$ a distribution with values in the space where $A$ is defined, and to the formulation and solution of an "abstract Goursat problem" that reduces to the abstract Cauchy problem when $n=1$.

Throughout this paper $E$ will be a quasi-complete, barreled (tonnele) linear topological space over the field $C$ of complex numbers ([1], Ch. II, $\S 4$; [2], Ch. III, $\S 1$ and $\S 2$ ), $A$ a closed linear operator with domain $D(A)$ dense in $E$ and range in $E$. For $n$ an integer $\geqq 1 R^{n}$ denotes $n$-dimensional Euclidean space endowed with its natural vector space operations; elements of $R^{n}$ will be written $s=\left(s_{1}, \cdots, s_{n}\right)$, $t=\left(t_{1}, \cdots, t_{n}\right)$, etc. We shall also denote $R_{+}^{n}$ (resp. $\left.\bar{R}_{+}^{n}\right)$ the subset of $R^{n}$ consisting of elements $t=\left(t_{1}, \cdots, t_{n}\right)$ with $t_{k}>0$ (resp. $t_{k} \geqq 0$ ), $1 \leqq k \leqq n$. The symbol $Z^{n}$ stands for the set of all points in $R^{n}$ with integer coordinates; we also define $Z_{+}^{n}=Z^{n} \cap R_{+}^{n}, \bar{Z}_{+}^{n}=Z^{n} \cap \bar{R}_{+}^{n}$. Elements in $Z_{+}^{n}, \bar{Z}_{+}^{n}$ will be denoted $\alpha=\left(\alpha_{1}, \cdots, \alpha_{n}\right), \beta=\left(\beta_{1}, \cdots, \beta_{n}\right)$, etc. Finally, for $\alpha \in \bar{Z}_{+}^{n}$ the symbol $D^{\alpha}$ means, as customary, the differential monomial $D_{1}^{\alpha_{1}} D_{2}^{\alpha_{2}} \cdots D_{n}^{\alpha_{n}}$, where $D_{k}=\partial / \partial t_{k}, 1 \leqq k \leqq n$.

The operator $A$ belongs to the class $\Theta(\alpha)$ if there exists a distribution $S_{\alpha}$ in the $n$ variables $t_{1}, \cdots, t_{n}$ with values continuous operators from $E$ into $D(A)$ (that is, a $S_{\alpha} \in \mathscr{D}^{\prime}\left(R^{n} ; \mathscr{L}(E, D(A))\right.$; see definitions in next section) locally of finite order, with support in $\bar{R}_{+}^{n}$, commuting with $A\left(A S(\varphi)=S(\varphi) A\right.$ for all test functions $\varphi$ in $\left.\mathscr{D}\left(R^{n}\right)\right)$ and satisfying

$$
\left(D^{\alpha}-A\right) S_{\alpha}=\delta \otimes I
$$

where $\delta$ is the Dirac measure in $R^{n}$. We relate in $\S 2$ the previous assumption on $A$ with solvability of the equation

$$
\left(D^{\alpha}-A\right) U=T
$$

where $T$ is an arbitrary distribution with values in $E$ and support in some translate of $\bar{R}_{+}^{n}$; this is done by reduction to the case of one variable $(n=1)$ where the results are well known ([3] for the case 
$\alpha=1$, [4] for $\alpha \geqq 1$ ). Section 3 is (mostly) devoted to the study of the case $|\alpha|=\alpha_{1}+\cdots+\alpha_{n} \geqq 3$, again by reduction to the case of one variable and-this time-by application of results in [6]. In particular, it is proved that $\Theta(\alpha)=\Theta(|\alpha|)$ for $|\alpha| \geqq 3$, which provides a complete identification of these classes (Recall that, according to [6], especially Theorem 4.1, the class $\Theta(\alpha)$ for $\alpha$ an integer $\geqq 3$. consists of those continuous, everywhere defined operators $A$ such that the series

$$
M_{\alpha}(t A)=\sum_{j=0}^{\infty} \frac{t^{j}}{(\alpha j) !} A^{j} u
$$

converges in $E$ for all $u \in E$ and $t \geqq 0$ or, equivalently, such that

$$
\lim _{j \rightarrow \infty} \frac{t^{j}}{(\alpha j) !} A^{j} u=0
$$

for all $u \in E, t \geqq 0$. In 34 the previous results are applied to the solution of the "usual" Goursat problem, that is, finding ordinary (smooth) solutions of the equation

$$
D^{\alpha} u=A u
$$

in $\bar{R}_{+}^{n}$, the value of the solution and of its normal derivatives up to a convenient order given in the boundary of $\bar{R}_{+}^{n}$. A few comments on nomenclature may be in order. The name "Goursat problem" is used in current literature mostly in the following context: to find solutions $u=u\left(t_{1}, t_{2}, \cdots, t_{n}, x_{1}, x_{2}, \cdots, x_{m}\right)$ of the partial differential equation

$$
D^{\alpha} u=f
$$

for $\left(t_{1}, \cdots, t_{n}\right) \in \bar{R}_{+}^{n}$, the values of $u$ arbitrarily prescribed in the boundary of $\bar{R}_{+}^{n}$ (Here $\alpha=(1,1, \cdots, 1)$ and $f$ depends on the variables, on $u$ and on some of its partial derivatives with respect to $t_{1}, \cdots, t_{n}$ and $x_{1}, \cdots x_{m}$; the order of any of those partials with respect to the $t$-variables should be less than $n$ ). If one allows only derivatives with respect to the $x$-variables to appear in the right-hand side of (1.4) and $f$ is independent of $t_{1}, \cdots, t_{n}$ and linear in these derivatives then the equation (1.4) can be written-at least formally-in the form (1.3). (Note, however, that we do not assume $\alpha=(1,1, \cdots, 1)$ in (1.3)). In this framework we are able to give an abstract version of a result of A. Friedman ([7], $\S 9$ and [8], Chapter 7) where as in the case outlined above $A$ is a partial differential operator in the "space" variables $x_{1}, \cdots, x_{m}$ and $E$ itself is a distribution space (note, incidentally, that the space $E$ in [7] is not a Banach space, which provides a justification for setting the abstract Goursat problem in 
spaces more general than Banach). Section 5 deals with the case $|\alpha|=2$-the case $|\alpha|=1$ is just the well-known first-order Cauchy problem - and completes the results in $\S 3$ by showing that $\Theta((1,1))=$ $\Theta(2)$. A similar, but weaker property is shown for the classes $\Xi(\alpha)$, defined with reference to the usual Goursat problem. The last section is devoted to the case in which $E$ is a Banach space and relies heavily on [3], where the case $n=1$ was considered. Although the results for $|\alpha| \geqq 3$ are merely particular cases of the previous ones for general $E$, a better characterization of the class $\Theta((1,1))$ is obtained.

Observe, finally, that when $A$ is a partial differential operator it is in general possible to define $E$, as a space of functions or distributions, in such a way as to obtain a Banach space-then $A$ is not continuous except for trivial cases-or to allow $E$ to be a more general space, but rendering $A$ continuous. Treating these two cases separately would probably allow for some simplification of detail but would also make for a lengthier exposition and a real loss of generality; thus, we have adopted a unified point of view.

The present paper can be considered an extension (or continuation) of [6]; nevertheless, no great familiarity with [6]-except for Theorem 4.1 -is required.

2. The Goursat problem for distributions and the classes $\Theta(\alpha)$. We shall assume $D(A)$, as customary, endowed with the strongest topology that makes the maps $u \rightarrow u$ and $u \rightarrow A u$ continuous. Under this topology $D(A)$ is a quasi-complete locally convex linear topological space. (If $E$ is a Banach space this topology is generated, for instance, by the norm $\left.|u|_{D(A)}=|u|_{E}+|A u|_{E}\right)$.

In the following remarks the spaces $F, G, \cdots$ are quasi-complete locally convex linear topological spaces. The space $\mathscr{L}(F, G)$ consists of all linear continuous operators from $F$ to $G$ endowed with the topology of uniform convergence on bounded sets of $F$. If $F$ is barreled then $\mathscr{L}(F, G)$ is a locally convex, quasi-complete linear topological space (see [2], Ch. III, 3, No. 7). Moreover, the "equicontinuity principle" holds in $\mathscr{L}(F, G)$, that is, the three following properties are equivalent for a family $\left\{B_{\nu}\right\} \subseteq \mathscr{C}(F, G)$; (a) $\left\{B_{\nu}\right\}$ is bounded (b) $\left\{B_{\nu}\right\}$ is equicontinuous (c) $\left\{B_{\nu} u\right\}$ is bounded in $G$ for each $u \in F([2]$, Ch. III, §̧ 3 , Théorème 2).

Modifying slightly a notation of [13] we shall call $\mathscr{D}\left(R^{n}\right)$ the space of all complex-valued infinitely difierentiable functions with compact support equipped with its usual L. Schwartz topology ([13], Ch. III). According to [13], p. 49, the space $\mathscr{D}^{\prime}\left(R^{n} ; F\right)$ of $F$-valued distributions (of $n$ variables) is $\mathscr{L}\left(\mathscr{D}\left(R^{n}\right) ; F\right)$. Given $a=\left(a_{1}, \cdots, a_{n}\right) \in$ $R^{n}$ denote by $\mathscr{D}_{a}^{\prime}\left(R^{n} ; F\right)$ the subspace of $\mathscr{D}^{\prime}\left(R^{n} ; F\right)$ consisting of all distributions with support in $\bar{R}_{+}^{n}(a)=\left\{t \in R^{n} ; t_{k} \geqq a_{k}, 1 \leqq k \leqq n\right\}=$ 
$\left\{t+a ; t \in \bar{R}_{+}^{n}\right\}$ endowed with the topology that it inherits from $\mathscr{D}^{\prime}\left(R^{n} ; F\right)$. If $\left\{a_{m}\right\}$ is a sequence in $R^{n}$ such that $\bigcap_{m=-\infty}^{\infty} \bar{R}_{+}^{n}\left(a_{m}\right)=\varnothing$ (for instance $\left.a_{m}=(m, m, \cdots, m)\right)$, then we write

$$
\overline{\mathscr{D}}_{+}^{\prime}\left(R^{n} ; F\right)=\bigcup_{m=-\infty}^{\infty} \mathscr{D}_{a_{m}}^{\prime}\left(R^{n} ; F\right)
$$

and we assign to $\overline{\mathscr{D}}_{+}^{\prime}\left(R^{n} ; F\right)$ the inductive limit of the topologies of the $\overline{\mathscr{D}}_{a}^{\prime}\left(R^{n} ; F\right)([1]$, Ch. II, $\S 4)$. As customary, we write $\mathscr{D}^{\prime}\left(R^{n} ; C\right)=$ $\mathscr{D}^{\prime}\left(R^{n}\right)$ and similarly for other distribution spaces. (For additional information on the above definitions see [13], [14], [15].)

If $\Omega$ is any open subset of $R^{n}$ we shall denote by $\mathscr{D}(\Omega)$ the subspace of $\mathscr{D}\left(R^{n}\right)$ consisting of all functions $\varphi \in \mathscr{D}\left(R^{n}\right)$ with support contained in $\Omega$, endowed with the topology that it inherits from $\mathscr{D}\left(R^{n}\right)$. As for $\mathscr{D}^{m}(\Omega)$ ( $m$ an integer $\geqq 0$ ), it consists of all functions $\varphi$ with support in $\Omega$ having continuous partials $D^{p} \varphi$ for all $p \in Z_{+}^{n}$ with $|p| \leqq m$; a sequence $\left\{\Phi_{\nu}(\cdot)\right\}$ converges to zero in $\mathscr{D}^{m}(\Omega)$ if and only if the supports of the $\varphi_{\nu}^{\prime} \mathrm{s}$ are contained in a fixed compact subset of $\Omega$ and $D^{p} \varphi_{\nu}(\cdot) \rightarrow 0$ uniformly for all $p$ with $|p| \leqq m$.

We shall say that a distribution $T \in \mathscr{D}^{\prime}\left(R^{n} ; F\right)$ has (or is of) finite order locally, if, given any open bounded set $\Omega$ there exists an integer $m$ (depending in general on $\Omega$ ) such that $T$ is a continuous operator from $\mathscr{D}(\Omega)$-endowed with the topology of $\mathscr{D}^{m}(\Omega)$ - to $F$. ([14], p. 83) or, equivalently, such that $T$ can be extended to a continuous operator from $\mathscr{D}^{m}(\Omega)$ to $F$ (we design again by $T$ the extension). The space of all such distributions will be denoted $\mathscr{D}^{\prime}\left(R^{n} ; F\right)_{f}$; similarly $\overline{\mathscr{D}}_{+}^{\prime}\left(R^{n} ; F\right)_{f}=\mathscr{D}^{\prime}\left(R^{n} ; F\right)_{f} \cap \overline{\mathscr{D}}_{+}^{\prime}\left(R^{n} ; F\right)$. (If $F$ is a Banach, or more generally a $(D F)$-space-definition in [15], p. 201-then $\mathscr{D}^{\prime}\left(R^{n}, F\right)_{f}=\mathscr{D}^{\prime}\left(R^{n} ; F\right)$ as shown in [14], p. 85. However, this is not true in general, as the example in [14], p. 83 shows.)

Distributions that are locally of finite order are (also locally) derivatives of continuous functions; see [14], Proposition 24, p. 86. We shall need several times in what follows a result of similar type, less general but yielding additional information about supports.

THEOREM 2.0. Let $T \in \mathscr{D}^{\prime}\left(R^{n} ; F\right)_{f}$ with support in $\bar{R}_{+}^{n}(a)$ for some $a=\left(a_{1}, \cdots, a_{n}\right) \in R^{n}$. Let $\left(b_{1}, \cdots, b_{n}\right),\left(c_{1}, \cdots, c_{n}\right) \in R^{n}$ be such that $b_{k}<a_{k}<c_{k}$ for $1 \leqq k \leqq n$. Finally, let

$$
\Omega=\left\{t ; b_{k}<t_{k}<c_{k} ; 1 \leqq k \leqq n\right\} .
$$

Then there exists a continuous, $F$-valued function $f$ defined in $\Omega$ and with support in $\Omega \cap \bar{R}_{+}^{n}(\alpha)$ and $a p \in Z_{+}^{n}$ such that

$$
T=D^{p} f
$$


in $\Omega$.

Proof. Let $\Omega^{\prime}$ be any bounded open set containing the closure of $\Omega, m$ the integer corresponding to $\Omega^{\prime}$ in the definition of finite order. Define

$$
\eta(t)=\frac{t_{1}^{m+1}}{(m+1) !} \cdot \frac{t_{2}^{m+1}}{(m+1) !} \cdots \frac{t_{n}^{m+1}}{(m+1) !}
$$

for $t \in \bar{R}_{+}^{n}, \eta(t)=0$ elsewhere; plainly, $\eta(\cdot)$ has continuous partial derivatives of all orders $\leqq m$ in $R^{n}$. Then, if $\chi(\cdot)$ is any function in $\mathscr{D}\left(\Omega^{\prime}\right)$ the function $s \rightarrow \chi(s) \eta(t-s)$ belongs to $\mathscr{D}^{m}\left(\Omega^{\prime}\right)$ for any $t$; moreover, the map $t \rightarrow \chi(\cdot) \eta(t-(\cdot))$ from $R^{n}$ to $\mathscr{D}^{m}\left(\Omega^{\prime}\right)$ is continuous. Then the $F$-valued map

$$
f(t)=T(\chi(\cdot) \eta(t-(\cdot)))
$$

is continuous in $R^{n}$; moreover, if $t_{k}<a_{k}$ for some $k$ the support of $\chi(\cdot) \eta(t-(\cdot))$ is disjoint from $\bar{R}_{+}^{n}(a)$, thus $f$ has support in $\bar{R}_{+}^{n}(a)$. Observe next that, is $\varphi$ if any function in $\mathscr{D}\left(R^{n}\right)$ we have

$$
\int_{R^{n}} \varphi(t) f(t) d t=T(\sigma),
$$

$\sigma$ the function (in $\mathscr{D}\left(R^{n}\right)$ ) defined by

$$
\sigma(s)=\chi(s) \int_{R^{n}} \eta(t-s) \varphi(t) d t
$$

where we have written $d t=d t_{1} \cdots d t_{n}$ (this follows easily approximating the integrals involved by means of Riemann sums). Assume now that $\chi(s)=1$ for $s \in \Omega$ and let $\varphi$ by any function in $\mathscr{D}(\Omega), p=$ $(m+2, \cdots, m+2)$. We have

$$
\left(D^{p} f\right)(\varphi)=(-1)^{|p|} \int_{R^{n}}\left(D^{p} \varphi(t) f(t) d t=T\left((-1)^{|p|} \psi\right),\right.
$$

where

$$
\psi(s)=\chi(s) \int_{R^{n}} \eta(t-s) D^{p} \varphi(t) d t=(-1)^{|p|} \chi(s) \varphi(s)=(-1)^{|p|} \varphi(s) .
$$

This shows that $D^{p} f=T$ in $\Omega$, as required.

It should be noted that the converse of Theorem 2.0 holds; more generally, if $T$ is a distribution in $\mathscr{D}^{\prime}\left(R^{n} ; F\right)$ and if for every open bounded set $\Omega \subset R^{n}$ there exists a continuous, $F$-valued function defined in $\Omega$ and a $p \in \bar{Z}_{+}^{n}$ such that (2.1) holds, then $T \in \mathscr{D}^{\prime}\left(R^{n} ; F\right)_{f}$. The proof is simple. 
We shall say that the Goursat problem for the equation

$$
\left(D^{\alpha}-A\right) U=T
$$

$\left(\alpha \in Z_{+}^{n}\right)$ is well set if (a) for every $T \in \mathscr{D}^{\prime}\left(R^{n} ; E\right)_{f}$ with support in some $\bar{R}_{+}^{n}(a), a \in R^{n}$ there exists a unique $U \in \overline{\mathscr{D}}_{+}^{\prime}\left(R^{n} ; D(A)\right)$ also with support in $\bar{R}_{+}^{n}(a)$ satisfying (2.2); (b) the map

$$
T \longrightarrow U=\mathscr{C l} T
$$

from $\overline{\mathscr{D}}_{+}^{\prime}\left(R^{n} ; E\right)_{f}$ to $\overline{\mathscr{D}}_{+}^{\prime}\left(R^{n} ; D(A)\right)$ defined by equation $(2.2)$-which map, by virtue of (a), is well defined and linear-is continuous. $\left(\overline{\mathscr{D}}_{+}^{\prime}\left(R^{n} ; E\right)_{f}\right.$ is here assumed to inherit the topology of $\left.\overline{\mathscr{D}}_{+}^{\prime}\left(R^{n} ; E\right)\right)$.

Observe that the uniqueness condition in (a) is only stated in reference to $\overline{\mathscr{D}}_{+}^{\prime}\left(R^{n} ; D(A)\right)$; that is, there may well be, say, nonnull distributions $U$ in $\mathscr{D}^{\prime}\left(R^{n} ; D(A)\right)$ such that $\left(D^{\alpha}-A\right) U=0$. As for (b), in view of the definition of the inductive limit topology, it can be stated as follows: if $\left\{T_{\nu}\right\}$ is a generalized sequence in $\mathscr{D}^{\prime}\left(R^{n} ; E\right)_{f}$ such that the supports of all the $T_{\nu}$ are contained in a fixed $\bar{R}_{+}^{n}(\alpha)$ and $T_{\nu} \rightarrow 0$, then the generalized sequence $\left\{U_{\nu}\right\}$ in $\mathscr{D}^{\prime}\left(R^{n} ; D(A)\right)$ provided by (2.2) converges to zero.

Let now $\varphi \in \mathscr{D}\left(R^{n}\right)$. Define a linear operator from $E$ to $D(A)$ by

$$
S_{\alpha}(\varphi) u=(\mathscr{C}(\delta \otimes u))(\varphi), \quad u \in E .
$$

If $\left\{u_{\nu}\right\}$ is a generalized sequence in $E$ such that $u_{\nu} \rightarrow 0$, then $\delta \otimes u_{\nu} \rightarrow 0$ in $\mathscr{D}^{\prime}\left(R^{n} ; E\right)$; continuity of $\mathscr{C l}$ shows that $S_{\alpha}(\varphi) u_{\nu} \rightarrow 0$ in $D(A)$. Accordingly, $S_{\alpha}(\varphi) \in \mathscr{L}(E, D(A))$. Assume now that $\left\{\varphi_{\nu}\right\}$ is a generalized sequence in $\mathscr{D}\left(R^{n}\right)$ such that $\varphi_{\nu} \rightarrow 0$ and let $K$ be a bounded set in $F$. Then $\{\delta \otimes u ; u \in K\}$ is a bounded set in $\overline{\mathscr{D}}_{+}^{\prime}\left(R^{n} ; E\right)$. Since $\mathscr{C l}$ is a continuous operator it takes bounded sets into bounded sets ([2], Ch. III, § 2.3) thus $\{\mathscr{C}(\delta \otimes u) ; u \in K\}$ is bounded in $\overline{\mathscr{D}}_{+}^{\prime}\left(R^{n} ; D(A)\right)$. Using now the fact that $\mathscr{D}\left(R^{n}\right)$ is a barreled space ([13], Ch. III, Théorème 2) and the equicontinuity principle formulated at the beginning of this section we see that $\{\mathscr{C}(\delta \otimes u), u \in K\}$ is equicontinuous (as a subset of $\mathscr{L}\left(\mathscr{D}\left(R^{n}\right) ; D(A)\right)$. But then $\lim S_{\alpha}\left(\varphi_{\nu}\right) u=\lim \mathscr{C l}(\delta \otimes u)\left(\varphi_{\nu}\right)=0$ uniformly for $u$ in $K$, which shows that $\varphi \rightarrow S_{\alpha}(\varphi)$ is a distribution in $\mathscr{D}^{\prime}\left(R^{n} ; \mathscr{L}(E, D(A))\right.$. It is clear from (2.4) that $S_{\alpha}$ satisfies

$$
\left(D^{\alpha}-A\right) S_{\alpha}=\delta \otimes I \text {. }
$$

On the other hand, if $\varphi \in \mathscr{D}$ is such that $\operatorname{supp}(\varphi) \cap \bar{R}_{+}^{n}=\varnothing$ (where $\operatorname{supp}(\varphi)$ denotes the support of $\varphi) S_{\alpha}(\varphi)=(\mathscr{L}(\delta \otimes u))(\varphi)=0$ - this follows from the fact that, according to (a) $\operatorname{supp}(\mathscr{C}(\delta \otimes u)) \subseteq \bar{R}_{+}^{n}-$ and thus $\operatorname{supp}\left(S_{\alpha}\right) \subseteq \bar{R}_{+}^{n}$. We shall call the distribution $S_{\alpha}$ the propagator or fundamental solution of the equation (2.2). Observe that, 
if $u \in D(A), \quad U_{1}=A S_{\alpha} u=D^{\alpha} S_{\alpha} u-\delta \otimes u, \quad U_{2}=S_{\alpha} A u$ then both distributions obviously belong to $\overline{\mathscr{D}}_{+}^{\prime}\left(R^{n} ; D(A)\right)$ and satisfy

$$
\left(D^{\alpha}-A\right) U_{j}=\delta \otimes u \text {. }
$$

Thus $U_{1}=U_{2}$, which shows that $S_{\alpha}$ and $A$ commute. Collecting all the results so far proved, one obtains the direct part of

Theorem 2.1. Assume that the Goursat problem for (3.1) is well set, and that the propagator $S_{\alpha}$ of (2.2) belongs to $\mathscr{D}^{\prime}\left(R^{n} ; \mathscr{L}(E, D(A))_{f}\right.$. Then $A \in \Theta(\alpha)$. Conversely, let $A \in \Theta(\alpha)$. Then the Goursat problem is well set and the propagator $S_{\alpha}$ of (2.2) (which satisfies (2.5) and then must coincide with the distribution $S_{\alpha}$ in the definition of the class $\Theta(\alpha))$ belongs to $\mathscr{D}^{\prime}\left(R^{n} ; \mathscr{L}(E, D(A))_{f}\right.$.

For the proof of the remaining half we shall need a few facts on convolutions of vector-valued distributions. Although they are very particular cases of results in [15] (especially Proposition 39, p. 167) an independent presentation seems reasonable, as they can be established by elementary means (moreover, some of the techniques sketched will also be used in $\lcm{3} 3)$.

Let $T \in \mathscr{D}^{\prime}\left(R^{n} ; F\right)$ and let $\varphi=\varphi(s, t)$ be a function in $\mathscr{D}\left(R^{2 n}\right)=$ $\mathscr{D}\left(R_{s}^{n} \times R_{t}^{n}\right)$ (the notation is self explanatory). Define an $F$-valued function of $s$ by applying $T$ to $\varphi(s, \cdot)$, that is

$$
\psi(s)=T(\varphi(s, \cdot)) \text {. }
$$

Plainly $\psi$ has compact support. Observe next that, if $h_{j}=$ $(0,0, \cdots, h, 0, \cdots, 0)$ ( $h$ in the $j$ th place $), 1 \leqq j \leqq n, h_{j}^{-1}\left(\varphi\left(s+h_{i}, \cdot\right)-\right.$ $\varphi(s, \cdot)) \rightarrow D_{j} \varphi(s, \cdot)$ for each $s$ in the topology of $\mathscr{D}\left(R_{t}^{2 n}\right)$. But then $D_{j} \psi$ exists and $D_{j} \psi(s)=T\left(\left(D_{j}\right)_{s} \varphi(s, \cdot)\right.$. Iterating this procedure, we see that $\psi$ has continuous partial derivatives of all orders and that, for any $q \in \bar{Z}_{+}^{n}$,

$$
\left(D^{q} \psi\right)(s)=T\left(D_{s}^{q} \varphi(s, \cdot)\right) .
$$

Let now $F$ be barreled, $S$ a distribution in $\mathscr{D}^{\prime}\left(R^{n} ; \mathscr{C}(F, G)\right)_{f}$ such that $\operatorname{supp}(S) \subseteq \bar{R}_{+}^{n}(a)$ for some $a \in R^{n}$. Pick $c=\left(c_{1}, \cdots, c_{n}\right) \in R_{+}^{n}$ such that

$$
\operatorname{supp}(\varphi) \subset \Omega \times \Omega
$$

where $\Omega=\left\{t ;\left|t_{k}-a_{k}\right|<c_{k}\right\}$ and let $f$ be a continuous, $\mathscr{L}(F, G)$ valued function with support in $\Omega \cap \bar{R}_{+}^{n}(a)$ such that

$$
S=D^{p} f \text { in } \Omega
$$

for some $p \in \bar{Z}_{+}^{n}$ (the existence of $f$ is assured by Theorem 2.0). Define 


$$
(S \odot T)(\varphi)=(-1)^{|p|} \int_{R^{n}} f(s)\left(D^{p} \psi\right)(s) d s
$$

where $\psi$ is the function given by (2.7). A few remarks are now in order.

I. Since $D^{p} \psi$ is continuous and has compact support the set $\left\{\left(D^{p} \psi\right)(s) ; s \in R^{n}\right\}$ is bounded in $G$. Boundedness of $\left\{\left(D^{p} \psi\right)(s)\right\}$ and continuity of $f$ imply continuity of the integrand in the right-hand side of (2.11), thus the integral exists.

II. Let $(\Omega, f, p),(\widetilde{\Omega}, \widetilde{f}, \widetilde{p})$ both satisfy the conditions (2.9) and (2.10). By repeated indefinite integration of $f, \widetilde{f}$ (if necessary) we may assume that $p=\widetilde{p}$. Then $D^{p}(f-\widetilde{f})=0$ in $\Omega_{0}=\Omega \cap \widetilde{\Omega}$; since $\operatorname{supp}(f-\widetilde{f}) \subseteq \bar{R}_{+}^{n}(a)$, this implies $f=\widetilde{f}$ in $\Omega_{0}$. Accordingly, Definition (2.11) is independent of $f, \Omega$ or $p$.

III. If $\left\{\varphi_{\nu}\right\}$ converges to zero in $\mathscr{D}\left(R^{2 n}\right)$ then, as the supports of all the $\varphi_{\nu}$ 's are contained in a fixed compact in $R^{2 n}$, it is clear that $f, \Omega$ and $p$ in the definition (3.11) can be chosen independently of $\nu$; on the other hand, it follows from $(2.8)$ that $D_{s}^{p} T(\varphi(s, \cdot)) \rightarrow 0$ uniformly for all $p$, thus $(S \odot T)\left(\varphi_{\nu}\right) \rightarrow 0$ in $G$. This shows that $S \odot T \in$ $\mathscr{D}^{\prime}\left(R^{2 n} ; G\right)$.

IV. It follows from the form of compact sets in $\mathscr{D}$ spaces that if a set $\mathscr{K}$ is compact in $\mathscr{D}\left(R^{2 n}\right)$ then so is $\left\{\varphi(s, \cdot) ; \varphi \in \mathscr{K}, s \in R_{s}^{n}\right\}$ in $\mathscr{D}\left(R_{t}^{n}\right)$. This shows that if $T_{\nu} \rightarrow 0$ in $\mathscr{D}^{\prime}\left(R^{n} ; F\right)$ then so does $S \odot T_{\nu}$ in $\mathscr{D}^{\prime}\left(R^{2 n} ; G\right)$.

V. It is easy to see that $\operatorname{supp}(S \odot T) \cong \operatorname{supp}(S) \times \operatorname{supp}(T)$; the proof is very similar to that for the scalar case, where $S \odot T$ coincides with the tensor product $S \otimes T$.

Assume now that $\operatorname{supp}(T) \subseteq \bar{R}_{+}^{n}(b)$ for some $b \in R^{n}$ and let $\varphi \in$ $\mathscr{D}\left(R^{n}\right)$. Denote by $\hat{\varphi}$ the function in $R^{2 n}=R_{s}^{n} \times R_{t}^{n}$ defined by $\hat{\varphi}(s, t)=\varphi(s+t)$, and let $\chi \in \mathscr{D}\left(R^{2 n}\right)$ such that $\chi=1$ in $\operatorname{supp}(\hat{\varphi}) \cap$ $\left(\bar{R}_{+}^{n}(a) \times \bar{R}_{+}^{n}(b)\right)$. Define

$$
(S * T)(\varphi)=(S \odot T)(\chi \widehat{\varphi})
$$

It is immediate from $V$ that Definition (2.12) is independent of $\chi$. Moreover if $\left\{\varphi_{\nu}\right\}$ converges to zero (resp. is bounded) in $\mathscr{D}\left(R^{n}\right)$ and $\left\{T_{\eta}\right\}$ is a set in $\mathscr{D}^{\prime}\left(R^{n} ; F\right)$ such that $\operatorname{supp}\left(T_{\eta}\right) \subseteq \bar{R}_{+}^{n}(a)$ for all $\eta$, then $\chi$ can be chosen independently of $\nu, \eta$ and $\chi \widehat{\varphi}_{\nu} \rightarrow 0$ (resp. $\left\{\chi \hat{\varphi}_{\nu}\right\}$ is bounded) in $\mathscr{D}^{\prime}\left(R^{2 n} ; F\right)$. Applying this observation with $\left\{T_{\eta}\right\}=\{T\}$ 
and $\varphi_{\nu} \rightarrow 0$ and using III we see that $S * T \in \mathscr{D}^{\prime}\left(R^{n} ; G\right)$. In a similar way, it follows from IV that the map

$$
T \rightarrow S * T
$$

from $\overline{\mathscr{D}}_{+}^{\prime}\left(R^{n} ; F\right)$ into $\overline{\mathscr{D}}_{+}^{\prime}\left(R^{n} ; G\right)$ is continuous.

Observe now that, since $D_{s}^{p}(S \odot T)=D_{s}^{p} \odot T$ for any $p \in \bar{Z}_{+}^{n}$ (immediate verification) it follows from (2.12) that

$$
\left(D_{s}^{p} S * T\right)(\varphi)=D_{s}^{p}(S \odot T)(\chi \widehat{\varphi})=(-1)^{|p|}(S \odot T)\left(D_{s}^{p}(\chi \widehat{\varphi})\right) .
$$

Noting now that $D_{s}^{p}(\chi \widehat{\varphi})=\chi D_{s}^{p} \widehat{\varphi}=\chi\left(\widehat{D^{p} \varphi}\right)$ in the subset of $R^{2 n}$ where $\chi=1$ we deduce

$$
D^{p}(S * T)=D^{p} S * T .
$$

Finally, it follows from $V$ that

$$
\operatorname{supp}(S * T) \subseteq \operatorname{supp}(S)+\operatorname{supp}(T) .
$$

We may note at this point the relation between the operations $\odot$ and * defined above and the definitions in [15]. According to Proposition 33 (especially equality II. $6 ; 2$ ) the product $S \odot T$ coincides with the image of the tensor product $S \otimes \otimes, T \in \mathscr{D}^{\prime}\left(R^{2 n} ; \mathscr{L}(F, G) \widehat{\otimes}_{\imath} F\right)$ by the canonical linear map $\pi: \mathscr{L}(F, G) \widehat{\otimes}_{,} F \rightarrow G$. Likewise, $S * T$ is the image under the same map of the convolution $S * T \in$ $\mathscr{D}^{\prime}\left(R^{n} ; \mathscr{L}(F, G) \widehat{\otimes}_{\imath} F\right)$ (see [15], Proposition 39, especially equality II. 7 ; 8; the definitions of the topological tensor products and maps involved can be found in the first pages of [15]).

These facts can now be applied to our problem as follows. Let $A \in \Theta(\alpha)$, and let $S_{\alpha} \in \mathscr{D}^{\prime}\left(R^{n} ; \mathscr{L}(E, D(A))\right)$ with support in $\bar{R}_{++}^{n}$ and satisfying

$$
\left(D^{\alpha}-A\right) S_{\alpha}=\delta \otimes I .
$$

Then, by virtue of (2.13), (2.14), (2.15) and surrounding comments, $S_{\alpha} * T$ furnishes a solution of $(2.2)$ for any $T \in \overline{\mathscr{D}}_{+}^{\prime}\left(R^{n} ; E\right)$ such that $\operatorname{supp}\left(S_{\alpha} * T\right) \subseteq \bar{R}_{+}^{n}(a)$ if $\operatorname{supp}(T) \subseteq \bar{R}_{+}^{n}(a)$. There only remains the question of uniqueness of this solution; that is, we have to show that if an $U \in \overline{\mathscr{D}}_{+}^{\prime}\left(R^{n} ; D(A)\right)$ satisfies

$$
\left(D^{\alpha}-A\right) U=0
$$

then $U=0$. We observe first that this needs to be shown only in the case $U$ is a $D(A)$-valued function, defined in $R^{n}$ and infinitely differentiable. For, if $\varphi \in \mathscr{D}\left(R^{n}\right), u=U * \varphi$ is such a function. By virtue of (2.14) $u$ satisfies (2.17), and it follows from (2.15) that $\operatorname{supp}(u)$ lies in some $\bar{R}_{+}^{n}(a)$. Then $u=0$; in particular $u(0)=(U * \varphi)(0)=$ 
$U(\breve{\varphi})=0$, where $\breve{\varphi}(s)=\varphi(-s)$. But $\varphi$-thus $\breve{\varphi}$-is arbitrary in $\mathscr{D}\left(R^{n}\right)$, hence $U=0$.

We settle next the case $n=1$ (one variable). To this end, let $u$ be as above; let $\varphi \in \mathscr{D}(R)$ with support in $t \leqq 0$ and define $\sigma=S_{\alpha} * \varphi$. Again $\sigma$ is an infinitely differentiable function-this time with values in $\mathscr{L}(E, D(A))$. In view of (2.16) it satisfies

$$
\left(D^{\alpha}-A\right) \sigma(t)=\varphi(t) I .
$$

Define now, for $t>0$

$$
v(s)=v(s, t, \varphi)=\sum_{j=0}^{a-1} \sigma^{(j)}(t-s) u^{(\alpha-1-j)}(a+s)
$$

where $a$ is such that $\operatorname{supp}(u)$ lies in $t \geqq a$. A moment's consideration shows that $v(\cdot)$ is infinitely differentiable: in particular,

$$
v^{\prime}(s)=\sigma(t-s) u^{(\alpha)}(\alpha+s)-\sigma^{(\alpha)}(t-s) u(\alpha+s) .
$$

Making now use of (2.17), (2.18) and of the fact that $\sigma(t)$ and $A$ commute for any $t$-a simple consequence of the property that $S$ and $A$ commute-we obtain

$$
v^{\prime}(s)=\varphi(t-s) u(a+s) .
$$

In particular, $v^{\prime}(s)=0$ for $s \leqq t$. Thus $v$ is constant for $s \leqq t$; as it vanishes for $s \leqq 0, v(s)=0$ for $s \leqq t$. Repeating this reasoning for any $t \geqq 0$ we obtain

$$
\sum_{j=0}^{\alpha-1} \sigma^{(j)}(0) u^{(\alpha-1-j)}(a+t)=0
$$

for $t \geqq 0$. Since $\sigma^{(j)}(0)=\left(S_{\alpha}^{(\jmath)} * \varphi\right)(0)=S^{(j)}(\ddot{\varphi})$, where $\breve{\varphi}(t)=\varphi(-t)$, it is clear that the fact that $u$ is everywhere zero will be a consequence of the following statement:

Let $u_{0}, \cdots, u_{\alpha-1} \in E$. Assume

$$
\sum_{j=0}^{\alpha-1} S_{\alpha}^{(j)}(\varphi) u_{j}=0
$$

for all $\varphi \in \mathscr{D}(R)$ with support in $t \geqq 0$. Then

$$
u_{0}=u_{1}=\cdots=u_{\alpha-1}=0 \text {. }
$$

Assume this is not true, and call $U=\sum_{j=0}^{\alpha \rightarrow 1} S_{\alpha}^{(j)} u_{j}$. Since $S_{\alpha}$ vanishes for $t<0$, so does $U$; on the other hand, (2.20) implies that $U$ vanishes as well for $t>0$. This and the fact that $S_{\alpha}$-thus $U$ is locally of finite order yields: either $U=0$ or 


$$
U=\sum_{k=0}^{m} \delta^{(k)} \otimes v_{k}
$$

for some $m \geqq 0$ and $v_{0}, \cdots, v_{m} \in E$. (See [6], §3 for a similar reasoning.) Making now use of (2.6) we see that $U$ must satisfy $\left(D^{\alpha}-A\right) U=$ $\sum_{j=0}^{\alpha-1} \delta^{(j)} \otimes u_{k}$ which, combined with (2.22) yields

$$
\sum_{k=0}^{m} \delta^{(k+\alpha)} \otimes v_{k}-\sum_{k=0}^{m} \delta^{(k)} \otimes A v_{k}=\sum_{j=0}^{\alpha-1} \delta^{(j)} \otimes A u_{j} \cdot
$$

Equating the coefficients of each derivative of $\delta$ it is plain that (2.22) implies (2.21).

We now proceed to the case $n>1$. Denote, as in [13], p. 43 by $Y_{\rho}$ ( $\rho$ any complex number) the distribution in $\mathscr{D}^{\prime}(R, C)$ defined by the function

$$
\frac{\left(h(t)(t)^{\rho-1}\right.}{\Gamma(\rho)}
$$

( $h$ the Heaviside function equal to 1 for $t \geqq 0$, 0 for $t<0$ ) when Re $\rho>0$ and extended to all values of $\rho$ by analytic continuation (see [13], Ch. II or [9], Ch. I where this distribution is denoted $\left.t_{+}^{o-1} / \Gamma(\rho)\right)$. For each $\rho$ the support of $Y_{\rho}$ lies in $t \geqq 0$; moreover, we have

$$
Y_{\rho_{1}} * Y_{\rho_{2}}=Y_{\rho_{1}+\rho_{2}}, \quad Y_{\rho}^{\prime}=Y_{\rho_{-1}}, \quad Y_{0}=\delta .
$$

Let now $\mathscr{E}=\mathscr{D}^{\prime}\left(R^{n-1} ; E\right)$. Define an operator $\mathscr{A}$ in this space as follows: $D(\mathscr{A})=\mathscr{D}^{\prime}\left(R^{n-1} ; D(A)\right)$ and for any $V \in D(\mathscr{A})$

$$
\mathscr{A} V=\left(Y_{\alpha_{1}} \otimes Y_{\alpha_{2}} \otimes \cdots \otimes Y_{\alpha_{n-1}}\right) * A V
$$

where each $Y_{\alpha_{k}}$ acts on the variable $t_{k}$ and $\otimes$ is the usual tensor product of distributions. It is easy to verify that $D(\mathscr{A})$ is dense and that $\mathscr{A}$ is closed. Take now the solution $S_{\alpha}$ of (2.16) and define, for $\varphi \in \mathscr{D}\left(R^{n-1}\right), \psi \in \mathscr{D}(R)$

$$
\begin{aligned}
& (\mathscr{S}(\psi))(\varphi)=\left(D_{1}^{\gamma_{1}} \cdots D_{n-1}^{\gamma_{n-1}} S_{\alpha}\right)(\varphi \otimes \psi) \\
= & {\left[\left(Y_{-\alpha_{1}} \otimes \cdots \otimes Y_{-\alpha_{n-1}}\right) * S_{\alpha}\right](\varphi \otimes \psi) }
\end{aligned}
$$

(in the last expression we have used the fact-plain from (2.23)that $Y_{-m}=\delta^{(m)}$ for any integer $m$ ). A moment's consideration shows that $\mathscr{S} \in \mathscr{D}^{\prime}(R ; D(\mathscr{A}))$. Moreover

$$
\begin{aligned}
& \left(\left(D^{\alpha_{n} \mathscr{S}}\right)(\psi)\right)(\varphi)=\left(\left((-1)^{\alpha_{n}} \mathscr{S}\right)\left(D^{\alpha_{n}} \psi\right)\right)(\varphi) \\
= & (-1)^{\alpha_{n}}\left(D_{1}^{\imath_{1}} \cdots D_{n}^{\gamma_{n-1} S_{\alpha}}\right)\left(D_{n}^{\gamma_{n}}(\varphi \otimes \psi)\right)=D^{\alpha} S^{\alpha}(\varphi \otimes \psi), \\
& \left((\mathscr{S} \mathscr{S})\left(\psi^{\prime}\right)\right)(\varphi) \\
= & {\left[\left(Y_{\alpha_{1}} \otimes \cdots \otimes Y_{\alpha_{n-1}}\right) *\left(Y_{-\alpha_{1}} \otimes \cdots \otimes Y_{-\alpha_{n-1}}\right) * A S_{\alpha}\right](\varphi \otimes \psi) } \\
= & {\left[\left(\left(Y_{\alpha_{1}} * Y_{-\alpha_{1}}\right) \otimes \cdots \otimes\left(Y_{\alpha_{n}} * Y_{-\alpha_{n}}\right)\right) * A S_{\alpha}\right](\varphi \otimes \psi)=A S_{\alpha}(\varphi \otimes \psi) }
\end{aligned}
$$


in view of (2.24), (2.25). Then, taking into account that $\delta \in \mathscr{D}^{\prime}\left(R^{n}\right)$ can be written $\delta_{1} \otimes \cdots \otimes \delta_{1}, \delta_{1} \in \mathscr{D}^{\prime}(R)$ and that the operator of convolution by $\delta_{1} \otimes \cdots \otimes \delta_{1}(n-1$ times $)$ is the identity operator $\mathscr{T}$ in $\mathscr{E}$ we see that $\mathscr{S}$ satisfies

$$
\mathscr{S}^{\left(\alpha_{n}\right)}-\mathscr{A} \mathscr{S}=\delta_{1} \otimes \mathscr{T} \text {. }
$$

After the (simple) verification that $\mathscr{S}$ and $\mathscr{A}$ commute, this means that $\mathscr{A} \in \Theta\left(\alpha_{n}\right)$. Let now $U \in \overline{\mathscr{D}}_{+}^{\prime}\left(R^{n} ; D(A)\right)$ satisfy (2.17). Define $\mathscr{U} \in \mathscr{D}^{\prime}(R ; D(\mathscr{A}))$ again by the formula

$$
(\mathscr{U}(\psi))(\varphi)=\left(D_{1}^{\alpha_{1}} \cdots D_{n-1}^{\alpha_{n}-1} \mathscr{U}\right)(\varphi \otimes \psi),
$$

$\varphi \in \mathscr{D}\left(R^{n-1}\right), \psi \in \mathscr{D}(R)$. Plainly $\operatorname{supp}(\mathscr{C})$ lies in $t \geqq a$ for some $a$; moreover, a few manipulations similar to the ones above show that $\mathscr{Q}$ satisfies

$$
\mathscr{U}^{\left(\alpha_{n}\right)}-\mathscr{A} \mathscr{U}=0 \text {. }
$$

By the uniqueness property, already proved for $n=1$, $\mathscr{C}=0$ (it should be pointed out that, although $\mathscr{E}=\mathscr{D}^{\prime}\left(R^{n-1} ; E\right)$ may not be barreled, this property was not used in the proof for the case $n=1$ ). Taking now into account the definition of $\mathscr{C}$ and the fact (proved in

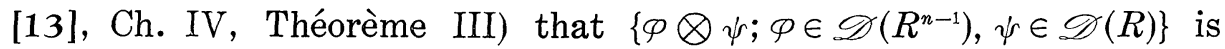
dense in $\mathscr{D}\left(R^{n}\right)$ we see that $D_{1}^{\gamma_{1}} \cdots D_{n-1}^{x_{n}-1} U=0$; taking advantage of the fact that $U \in \mathscr{D}_{+}^{\prime}\left(R^{n} ; D(A)\right)$ to convolute with $Y_{\alpha_{1}} \otimes \cdots \otimes Y_{\alpha_{n-1}}$ we obtain $U=0$, as desired. This ends the proof of Theorem 2.1.

3. The case $|\alpha| \geqq 3$. If $\beta=\left(\beta_{1}, \cdots, \beta_{n}\right), \gamma=\left(\gamma_{1}, \cdots, \gamma_{n}\right)$ are elements of $Z_{+}^{n}$ we shall write, for the sake of brevity, $\beta+\gamma=$ $\left(\beta_{1}+\gamma_{1}, \cdots, \beta_{n}+\gamma_{n}\right), \beta !=\beta_{1} ! \cdots \beta_{n} !, \beta j=\left(\beta_{1} j, \cdots, \beta_{n} j\right)$ for $j \in Z_{+}$; we will also write $1=(1,1, \cdots, 1)$ when there is no danger of confusion.

Given $\alpha \in Z_{+}^{n}$ we define the class $\Phi(\alpha) \subseteq \mathscr{L}(E, E)$ as follows; $A \in \Phi(\alpha)$ if and only if it is continuous, everywhere defined and

$$
\lim _{j \rightarrow \infty} \frac{r^{j}}{(\alpha j) !} A^{j} u=0
$$

for all $u \in E, r>0$. As it will be immediately seen, $\Phi(\alpha)$ depends only on $|\alpha|=\alpha_{1}+\cdots+\alpha_{n}$.

Lemma 4.1. For every $\alpha \in Z_{+}^{n}, \Phi(\alpha)=\Phi(|\alpha|)$.

Proof. Clearly

$$
(\alpha j) ! \leqq(|\alpha| j) !, \quad j \geqq 1
$$


thus

$$
\Phi(\alpha) \subseteq \Phi(|\alpha|)
$$

for any $\alpha \in Z_{+}^{n}$. On the other hand, according to Stirling's asymptotic formula ([10], formula 8.327),

$$
\begin{aligned}
(\alpha j) ! & =\prod_{k=1}^{n} \Gamma\left(\alpha_{k} j+1\right)=(2 \pi)^{n / 2} \prod_{k=1}^{n}\left(\alpha_{k} j\right)^{\alpha_{k} j+1 / 2} e^{-\alpha_{k} j}(1+o(1)) \\
& \geqq(2 \pi)^{n / 2} j^{|\alpha| j+n / 2} e^{-|\alpha| j}(1+o(1))
\end{aligned}
$$

as $j \rightarrow \infty$. Another application of Stirling's formula yields

$$
(|\alpha| j) !=|\alpha| j \Gamma(|\alpha| j)=(2 \pi)^{1 / 2}(|\alpha| j)^{|\alpha| j+1 / 2} e^{-|\alpha| j}(1+o(1))
$$

as $j>\infty$. Combining (3.2) and (3.3) we see that there exists a constant $\rho>0$ such that

$$
(\alpha j) !>\rho^{j}(|\alpha| j) !
$$

for $j$ large enough, which proves that

$$
\Phi(|\alpha|) \subseteq \Phi(\alpha)
$$

thus ending the demonstration of the lemma.

We remark that, if $0<\alpha<\beta\left(\alpha, \beta \in Z_{+}\right)$,

$$
\Phi(\alpha) \subseteq \Phi(\beta) \text {. }
$$

The reverse inclusion is in general false, as the example in [6], $\S 5$ shows. If $E$ is a Banach space, however, we plainly have $\Phi(\alpha)=$ $\mathscr{L}(E, E)$ for any $\alpha \in Z_{+}^{n}$.

THEOREM 3.2. Let $\alpha \in Z_{+}^{n},|\alpha| \geqq 3$. Then $\Theta(\alpha)=\Phi(\alpha) \quad$ (in particular, $\Theta(\alpha) \subseteq \mathscr{L}(E, E))$. Moreover, $S_{\alpha}$-the solution in $\mathscr{D}^{\prime}\left(R^{n} ; \mathscr{L}(E, D(A))_{f}\right.$ of

$$
\left(D^{\alpha}-A\right) S_{\alpha}=\delta \otimes I
$$

commuting with $A$ and with support in $\bar{R}_{+}^{n}$-coincides there with a $\mathscr{L}(E, E)$-valued entire function.

Proof. Assume $A \in \Phi(|\alpha|)=\Phi(\alpha)$. It is a consequence of (3.1) that, for any $r>0$ the set $\left\{r^{j}((\alpha j) !)^{-1} A^{j} u, j=1,2, \cdots\right\}$ is bounded in $E$ for any $u \in E$. But then it follows from the equi-continuity principle in $\S 2$ that $\left\{r^{j}((\alpha j) !)^{-1} A^{j}, j=1,2, \cdots\right\}$ is bounded in $\mathscr{L}(E, E)$. Due to the arbitrariness of $r$ this means that 


$$
\lim _{j \rightarrow \infty} \frac{r^{j}}{(\alpha j) !} A^{j}=0
$$

in the topology of $\mathscr{L}(E, E)$. After some simple manipulations we see that (3.5) implies that the series

$$
\sum_{j=0}^{\infty} \frac{\zeta^{j}}{(\alpha j+\alpha-1) !} A^{j} u
$$

is convergent in $\mathscr{L}(E, E)$, uniformly for $\zeta$ on compact subsets of $C$, thus it defines an entire function $B(\zeta)$ with values in $\mathscr{L}(E, E)$.

Let now $t=\left(t_{1}, \cdots, t_{n}\right) \in R^{n}$; for $\beta \in \bar{Z}_{+}^{n}$ write $t^{\beta}=t_{1}^{\beta_{1}} t_{2}^{\beta_{2}} \cdots t_{n}^{\beta_{n}}$. Define $S_{\alpha}(t)=t^{\alpha-1} B\left(t^{\alpha}\right)$ if $t \in \bar{R}_{+}^{n}$, =0 elsewhere. Making use of the distributions $Y_{\rho}$ (see preceding section) it is clear that $S_{\alpha}$, as an element of $\mathscr{D}^{\prime}\left(R^{n} ; \mathscr{L}(E, E)\right)$ can be written

$$
S_{\alpha}=\sum_{j=1}^{\infty}\left(Y_{\alpha_{1} j} \otimes \cdots \otimes Y_{\alpha_{n} j}\right) \otimes A^{j}
$$

where the series, by virtue of the comments following (3.6) is convergent in $\mathscr{D}^{\prime}\left(R^{n} ; \mathscr{L}(E, E)\right)$ and can thus be differentiated term by term any number of times. Doing this, using formula (2.23) and the possibility of writing $\delta=\delta_{1} \otimes \cdots \otimes \delta_{1}(n$ times $), \delta_{1}$ the Dirac measure in $R$, we obtain after a simple computation

$$
\left(D^{\alpha}-A\right) S_{\alpha}=\delta \otimes I \text {. }
$$

On the other hand it is plain from (3.7) that $S_{\alpha}$ and $A$ commute. Thus

$$
\Phi(\alpha) \subseteq \Theta(\alpha)
$$

(observe that (3.8) has actually been proved for all $\alpha \in Z_{+}^{n}$, a result of some interest for $|\alpha|<3$ as well). We now proceed to the proof of the reverse inclusion for $|\alpha| \geqq 3$. Let $T$ be a distribution in some $\mathscr{D}^{\prime}\left(R^{n} ; F\right)(F$ as in $\S 2$ an arbitrary quasi-complete locally convex linear topological space) with support in some $\bar{R}_{+}^{n}(a)$. We shall obtain by means of $T$ a new distribution $\widetilde{T} \in \mathscr{D}_{+}^{\prime}(R ; F)$-which might be called the "autoconvolution" of $T$-as follows. Let $\varphi \in \mathscr{X}(R)$ and define

$$
\widetilde{T}(\varphi)=T(\chi \hat{\varphi})
$$

where

$$
\widehat{\varphi}\left(t_{1}, \cdots, t_{n}\right)=\varphi\left(t_{1}+\cdots+t_{n}\right)
$$

and $\chi$ is any function in $\mathscr{D}\left(R^{n}\right)$ which equals 1 in $\bar{R}_{+}^{n}(a) \cap \operatorname{supp}(\widehat{\varphi})$. Exactly in the same way as in the definition of convolution in $\S 2$ it 
can be shown that (3.10) is independent of the particular $\chi$ used, that $\widetilde{T}$ belongs in fact to $\mathscr{D}_{+}^{\prime}(R ; F)$ and that, for any $p \in \bar{Z}_{+}^{n}$, $\varphi \in \mathscr{D}(R)$

$$
D^{|p|} \widetilde{T}(\varphi)=D^{p} T(\chi \widehat{\varphi})
$$

Observe that in the case $T$ coincides with a (say, continuous) function in $\sum s_{k}<b$ then $\widetilde{T}$ is as well a continuous function in $t<b$ given by the formula

$$
\widetilde{T}(t)=\frac{1}{\sqrt{n}} \int_{\Sigma s_{k}=t} T(s) d \sigma
$$

where $d \sigma$ is the element of (hyper) area of the hyperplane $\sum s_{k}=t$.

Assume then that $A \in \Theta(\alpha)$. Let $S_{\alpha}$ be the distribution in $\mathscr{D}^{\prime}\left(R^{n} ; \mathscr{L}(E, D(A))\right.$ satisfying (3.8) and commuting with $A$ and let $S_{|\alpha|}=\widetilde{S}_{\alpha}$. Plainly $S_{|\alpha|} \in \mathscr{D}^{\prime}(R ; \mathscr{L}(E, D(A))$, commutes with $A$ and its support is contained in $t \geqq 0$. Moreover, it follows easily from its definition that $S_{|\alpha|}$ is locally of finite order if $S_{\alpha}$ is. Applying the (evidently linear) transformation $\sim$ to both sides of the equality (3.8) and making use of the simple fact that $\tilde{\delta}=\delta_{1}$ we obtain

$$
\left(D^{|\alpha|}-A\right) S_{|\alpha|}=\delta_{1} \otimes I \text {. }
$$

This shows that $A \in \Theta(|\alpha|)$; in general,

$$
\Theta(\alpha) \subseteq \Theta(|\alpha|)
$$

(observe again that (3.13) has been established for all $\alpha \in Z_{+}^{n}$; an application for $|\alpha|=2$ will be found in $\S 5$ ). Returning to the case $|\alpha| \geqq 3$ note that (3.13) and Theorem 2.1 imply that the Goursat (or Cauchy) problem for

$$
\left(D^{|\alpha|}-A\right) U=T
$$

is well set. Making use of the results in [6] (specifically, of Theorem 4.1), we readily obtain that $A \in \Theta(|\alpha|)$, which ends the proof of Theorem 3.2.

REMARK 3.3. It should be noted that some of the results in this section admit of extensions to differential operators more general than the monomial $D^{\alpha}$. We limit ourselves to sketch one of them. Let $m \geqq 3$ be an integer, $P$ a homogenous differential polynomial of degree $m$, that is

$$
P=\sum_{|\alpha|=m} a_{\alpha} D^{\alpha}
$$


where the (complex) coefficients $a_{\alpha}$ are constant and

$$
\sum_{|\alpha|=m} a_{\alpha} \neq 0 \text {. }
$$

Assume that there exists a solution $S \in \mathscr{D}^{\prime}\left(R^{n} ; \mathscr{L}(E, D(A))_{f}\right.$ of the equation

$$
(P-A) S=\delta \otimes I
$$

with support in $\bar{R}_{+}^{n}$ and commuting with $A$. Then $A \in \Phi(m)$. The proof, as the one for the particular case $P=D^{\alpha}$ is based on formula (3.11).

REMARK 3.4. The Goursat problem (for partial differential operators) was studied by A. Friedman in [7], $\$ 6$ and [8], Chapter 7. Specifically, he considers the problem of existence and uniqueness of solutions $u=u(t, x)=u\left(t_{1}, \cdots, t_{m}, x_{1}, \cdots, x_{m}\right)$ of the partial differential equation

$$
D_{t}^{(1)} u(x, t)=P\left(i D_{k, x}\right) u(t, x)
$$

( $P$ a polinomial with constant coefficients, $1=(1,1, \cdots, 1)$ ) in $\bar{R}_{+}^{n}$ or, more generally, in a subset of $\vec{R}_{+}^{n}$, the values of $u$ having been prescribed in the boundary of $\bar{R}_{+}^{n}$, that is

$$
u\left(\left\langle t_{k}\right\rangle\right)=\eta_{k}\left((t)_{k}\right)
$$

where we have set $\left\langle t_{k}\right\rangle=\left(t_{1}, \cdots, t_{k-1}, 0, t_{k+1}, \cdots, t_{n}\right), \quad\left(t_{k}\right)=$ $\left(t_{1}, \cdots, t_{k-1}, t_{k+1}, \cdots, t_{n}\right), 1 \leqq k \leqq n$ and $\eta_{1}, \cdots, \eta_{n}$ are given in advance (see the next $\S 4$ for the relation between this type of problem and the abstract Goursat problem). Here $u$-as a "function" of $x$-is a distribution in a $W^{\prime}$ space (see definitions in [7], especially $\S 1$ ). An abstract version of (3.14) is also considered, although the definition of solution is different from ours. There is a result in [7], however, that is related to Theorem 3.2. In fact, a condition that insures solvability of (3.14), (3.15) ([7], condition 6.19) is seen not to hold, except for trivial cases when $n$, the number of variables, is $\geqq 3$; on the other hand, uniqueness of solutions of (3.14), (3.15) and continuous dependence on the $\eta_{k}$ 's are established under conditions more general than the ones demanded for existence. (See the remarks in [7], p. 885.)

4. The strong Goursat problem. We relate in this section the preceding results with the "usual" Goursat problem, i.e., that of solving the homogenous equation $D^{\alpha} u(t)=A u(t)$ in $\bar{R}_{+}^{n}$, the values of all derivatives $D^{\beta} u$ with $\beta_{k} \leqq \alpha_{k}-1,1 \leqq k \leqq n$ having been prescribed at the boundary of $\bar{R}_{+}^{n}$. 
A few more definitions and notations will now be necessary. If $\beta=\left(\beta_{1}, \cdots, \beta_{n}\right), \gamma=\left(\gamma_{1}, \cdots, \gamma_{n}\right)$ are elements of $\bar{Z}_{+}^{n}$ we write $\beta \leqq \gamma$ if $\beta_{1} \leqq \gamma_{1}, \cdots, \beta_{n} \leqq \gamma_{n}$. As in the previous section, $1=(1,1, \cdots, 1)$ when there is no danger of confusion. Finally, for any given $\alpha \in \bar{Z}_{+}^{n}$, $F$ as in $\S \S 2$ and $3, C^{(\alpha)}\left(\bar{R}_{+}^{n} ; F\right)$ denotes the space of all $F$-valued functions $u(\cdot)$ defined in $\bar{R}_{+}^{n}$ and having there continuous partial derivatives $D^{\beta} u(t)$ for $0 \leqq \beta \leqq \alpha$ (we note that, at the boundary of $\bar{R}_{+}^{n}$ the derivatives involving the "perpendicular" variable are onesided).

Let $\alpha \in Z_{+}^{n}$. The operator $A$ is said to belong to the class $\Xi(\alpha)$ if it belongs to $\Theta(\alpha)$ and, in addition, the solution of

$$
\left(D^{\alpha}-A\right) S_{\alpha}=\delta \otimes I
$$

commuting with $A$ and with support in $\bar{R}_{+}^{n}$ coincides there with a $\mathscr{L}(E, E)$-valued function $t \rightarrow S_{\alpha}(t)$ such that, for each $u \in E S_{\alpha}(\cdot) u \in$ $C^{(\alpha-1)}\left(\bar{R}_{+}^{n} ; E\right)$ (note that we do not assume $S_{\alpha}(\cdot)$ to belong to $\left.C^{(\alpha-1)}\left(\bar{R}_{+}^{n} ; \mathscr{L}(E, E)\right)\right)$. Clearly

$$
\Xi(\alpha) \subseteq \Theta(\alpha)
$$

for all $\alpha \in \bar{Z}_{+}^{n}$. On the other hand, it was shown in $\S 3$ that, when $|\alpha| \geqq 3$ any solution of (4.1) in $\mathscr{D}_{\div}^{\prime}\left(R^{n} ; \mathscr{L}(E, D(A))_{f}\right.$ commuting with $A$ and with support in $\bar{R}^{n}$ must of necessity be an $\mathscr{L}(E, E)$ valued holomorphic function there. Accordingly,

$$
\Xi(\alpha)=\Theta(\alpha)
$$

for $|\alpha| \geqq 3$. Thus the previous definition of $\Xi(\alpha)$ introduces something new only in the three cases $\alpha=1,2,(1,1)$. The first two cases (one variable) correspond to the Cauchy problem and can essentially be read off from the results in [4], [5], thus will not be treated here. As for the case $|\alpha| \geqq 3$-as pointed out in $\S 3$-the class $\Xi(\alpha)=\Theta(\alpha)$ is perhaps too restricted to include (say, differential) operators of interest in practice. Nevertheless a sketch of the available results is included at the end of the present section, after the case $\alpha=(1,1)$ has been treated in detail and then applied to a problem considered by Friedman in [7] and [8].

We begin by stating a well-known result which will be applied in the sequel.

LEMMA 4.0. Let $Q$ be a closed operator in E with domain $D(Q), f(\cdot)$ a function defined in the domain $J$ in $R^{n}$, with values in $D(Q)$ and such that $f(\cdot), Q f(\cdot)$ are continuous and integrable in $J$. Then $f=\int_{J} f(t) d t \in D(Q)$ and 


$$
Q f=\int_{J} Q f(t) d t
$$

The proof can be immediately achieved by approximating the two integrals involved by Riemann sums and using the closedness of $Q$.

Let $\eta_{1}, \eta_{2}$ be two functions defined in $\bar{R}_{+}$. We shall say that the pair $\left\{\eta_{1}, \eta_{2}\right\}$ is compatible if

$$
\eta_{1}(0)=\eta_{2}(0)
$$

Theorem 4.1. Assume $A \in \Xi((1,1))$.

(a) Let $\left\{\eta_{1}, \eta_{2}\right\}$ be a compatible pair of functions in $C^{(1)}\left(\bar{R}_{+}^{1} ; E\right)$ such that $\eta_{1}\left(t_{1}\right), \eta_{1}^{\prime}\left(t_{1}\right), \eta_{2}\left(t_{2}\right), \eta_{2}^{\prime}\left(t_{2}\right)$ belong to $D(A)$ for all $t_{1}, t_{2} \geqq 0$. Assume, moreover, that $A \eta_{1}^{\prime}(\cdot), A \eta_{2}^{\prime}(\cdot)$ are continuous in $\bar{R}_{+}$. Then there exists a strong solution $u(t)=u\left(t_{1}, t_{2}\right)$ of

$$
D_{1} D_{2} u(t)-A u(t)=0
$$

(that is, a $u \in C^{((1,1))}\left(\bar{R}_{+}^{2} ; E\right)$ such that $u(t) \in D(A)$ and satisfying (4.4) everywhere in $\bar{R}_{+}^{2}$ ) such that

$$
u\left(t_{1}, 0\right)=\eta_{1}\left(t_{1}\right), \quad u\left(0, t_{2}\right)=\eta_{2}\left(t_{2}\right)
$$

for $t_{1}, t_{2} \geqq 0$.

(b) Let $\left\{u_{\nu}(\cdot)\right\}$ be a generalized sequence of strong solutions of (4.4) such that $u_{\nu}(\cdot, 0), D_{1} u_{\nu}(\cdot, 0), u_{\nu}(0, \cdot), D_{2} u_{\nu}(0, \cdot) \rightarrow 0$ uniformly on compacts of $\bar{R}_{+\cdot}^{1}$. Then $u_{\nu}(\cdot) \rightarrow 0$ uniformly on compacts of $\bar{R}_{+}^{2}$.

Proof. Let $\left\{\varphi_{n}\right\}$ be a " $\delta$-sequence" in $\mathscr{D}\left(R^{n}\right)$, that is a sequence such that $\varphi_{n} \geqq 0, \int \varphi_{n} d s=1$, diam $\operatorname{supp}\left(\varphi_{n}\right) \rightarrow 0$ and let $\left(\varphi_{n}\right)_{t}(s)=$ $\varphi_{n}(t-s)$. Since $S_{\alpha}$-as a distribution-commutes with $A$ we have, for any $t \in R_{+}^{n}, u \in D(A)$

$$
A \int\left(\varphi_{n}\right)_{t} S_{\alpha} u d s=\int\left(\varphi_{n}\right)_{t} S_{\alpha} A u d s
$$

Letting now $n \rightarrow \infty$ and using the smoothness of $S_{\alpha}$ and the closedness of $A$ we obtain that $S_{\alpha}(\cdot)$-which will be called simply $S(\cdot)$ from now on-commutes with $A$ as a function, i.e., $S(t) u \in D(A)$ for $u \in D(A), t \in R_{+}^{n}$ and

$$
A S(t) u=S(t) A u
$$

Equality (4.6) can actually be extended for all $t \in \bar{R}_{+}^{n}$, again because of the facts that $S(\cdot) \in C^{((0,0))}\left(\bar{R}_{+}^{2} ; E\right)$ and that $A$ is closed.

We need now to compute the values of $S$ at the boundary of $\bar{R}_{+}^{2}$. To this end, we write the equation 


$$
D_{1} D_{2} S u-A S u=\left(\delta_{1} \otimes \delta_{1}\right) \otimes u
$$

satisfied by $S u$ ( $u$ an element of $D(A), \delta_{1}$ the 1-dimensional Dirac measure) in the form

$$
D_{1} D_{2}[S u-(h \otimes h) * A S u]=\left(\delta_{1} \otimes \delta_{1}\right) \otimes u,
$$

where $h$ is the Heaviside function. Convoluting both sides of (4.7) by $h \otimes h=Y_{1} \otimes Y_{1}$ and making use of (2.23) we obtain

$$
S u-(h \otimes h) * A S u=(h \otimes h) \otimes u .
$$

Now, since both sides of (4.8) are continuous functions of $t$ in $\bar{R}_{+}^{2}-$ the left-hand side because of (4.6) and preceding comments - the equality must hold pointwise, which clearly implies $S\left(t_{1}, 0\right) u=S\left(0, t_{2}\right) u=$ $u$; since $D(A)$ is dense in $E$,

$$
S\left(t_{1}, 0\right)=S\left(0, t_{2}\right)=I
$$

for $t_{1}, t_{2} \geqq 0$.

We define a function $u$ in $\bar{R}_{+}^{2}$ by the formula

$$
\begin{aligned}
u\left(t_{1}, t_{2}\right)=S\left(t_{1}, t_{2}\right) \eta_{1}(0) & +\int_{0}^{t_{1}} S\left(t_{1}-s_{1}, t_{2}\right) \eta_{1}^{\prime}\left(s_{1}\right) d s_{1} \\
& +\int_{0}^{t_{2}} S\left(t_{1}, t_{2}-s_{2}\right) \eta_{2}^{\prime}\left(s_{2}\right) d s_{2} .
\end{aligned}
$$

It is easy to show that $u(\cdot)$ is continuous in $\bar{R}_{+}^{2}$; equality (4.9) and the compatibility condition (4.3) imply that $u(\cdot)$ satisfies the initial conditions (4.5). Observe next that making use of (4.10) and of Lemma 4.0 we obtain that $u(t) \in D(A)$ for all $t \in \bar{R}_{+}^{2}$ and $A u(\cdot)$ is continuous in $\bar{R}_{+}^{2}$.

It is plain that $u$ is none other than the convolution of $S$ by the distribution

$$
T=\left(\delta_{1} \otimes \delta_{1}\right) \otimes \eta_{1}(0)+\eta_{1}^{\prime} \otimes \delta_{1}+\delta_{1} \otimes \eta_{2}^{\prime} \in \mathscr{D}^{\prime}\left(R^{2} ; E\right)_{f}
$$

(where we have identified $\eta_{1}^{\prime}, \eta_{2}^{\prime}$ with the distributions they define) and, consequently, $u$-extended to the complement of $\bar{R}_{+}^{2}$ by setting it equal to zero there-satisfies

$$
\left(D_{1} D_{2}-A\right) u=T
$$

in the sense of distributions. A reasoning rather similar to the one yielding (4.7) applies now to show that

$$
\begin{aligned}
u=(h \otimes h) *(A u) & +(h \otimes h) \otimes \eta_{1}(0) \\
& +\left(\eta_{1}-\eta_{1}(0)\right) \otimes h+h \otimes\left(\eta_{2}-\eta_{2}(0)\right)
\end{aligned}
$$


which immediately shows that $u(\cdot) \in C^{((1,1))}\left(\bar{R}_{+}^{2} ; E\right)$. Since $\operatorname{supp}(T)$ is contained in the boundary of $\bar{R}_{+}^{2}$, $u$ must satisfy (4.4) in $R_{+}^{2}$-thus in $\bar{R}_{+}^{2}$ which ends the proof of (a) in Theorem 4.1. We shall prove (b) by showing that the formula (4.10) is valid for any strong solution $v(\cdot)$ of $(4.4)$-with $\eta_{1}\left(t_{1}\right)=v\left(t_{1}, 0\right), \eta_{2}\left(t_{2}\right)=v\left(0, t_{2}\right)$-even if the assumptions on $\eta_{1}, \eta_{2}$ in (a) are not satisfied. Let then $v$ be a strong solution of (4.4). Extend it to the complement of $\bar{R}_{+}^{2}$ as being zero there. A short computation shows that $v$ satisfies (4.12) in the sense of distributions, with $T$ given by (4.11). But it has been shown during the proof of (a) that $u$-defined by (4.10) in $\bar{R}_{+}^{2}$ and extended in the same way as $v$ to $R^{2}$-satisfies the same equation, also in the sense of distributions. By uniqueness of solutions of the Goursat problem (Theorem 2.1) $u=v$ as distributions; since both $u$ and $v$ are continuous functions in $\bar{R}_{+}^{2}, u(t)=v(t)$ for all $t \in \bar{R}_{+}^{2}$. Clearly, formula (4.10) yields (b), which ends the proof of Theorem 4.1.

REMARK 4.2. Let $A$ be a closed operator in $E$ such that $R(\lambda ; A)=$ $(\lambda I-A)^{-1}$ exists and is continuous for some $\lambda \in C$. Assume (a), (b) in Theorem 4.1 are satisfied. Then it is possible to show that $A \in \Xi((1,1))$, which is a sort of converse of that theorem. The proof-long but simple-runs much like the one for the Cauchy problem $(n=1)$ given in [11] and is therefore omitted. We only note that the propagator $S(\cdot)$ is constructed as follows: for $u \in D(A)$, $t \in \bar{R}_{+}^{2}$ define

$$
S(t) u=u(t)
$$

where $u(\cdot)$ is the solution of (4.4) with $u\left(t_{1}, 0\right)=u\left(0, t_{2}\right)=u$ and extend $S(\cdot)$ to all of $E$ by continuity.

REMARK 4.3. The results for the case $\alpha=(1,1)$ can be extended to any $\alpha=\left(\alpha_{1}, \cdots, \alpha_{n}\right),|\alpha| \geqq 3$. This extension, however, seems of limited interest, since for these values of $\alpha$ the classes $\Xi(\alpha)=\Theta(\alpha)=$ $\Phi(\alpha)$ are probably too restricted to include any (say, differential) operator of interest in applications. The "initial values" of a solution are now a family $\Phi=\left\{\eta_{\beta, k}\right\}$ of functions defined in $\bar{R}_{+}^{n-1}, 0 \leqq \beta \leqq \alpha-1$, $1 \leqq k \leqq n$ (the family $\Phi$ consists of $n \prod_{k=1}^{n} \alpha_{k}$ functions); we try to solve

$$
D^{\alpha} u(t)-A u(t)=0
$$

in $\bar{R}_{+}^{n}$, with initial conditions

$$
D^{3} u\left(\langle t\rangle_{k}\right)=\eta_{\beta, k}\left((t)_{k}\right)
$$

for $t \in \bar{R}_{+}^{n}, 0 \leqq \beta \leqq \alpha-1$, where we have set, for any $t=\left(t_{1}, t_{2}, \cdots, t_{n}\right) \in$ 
$R^{n},\langle t\rangle_{k}=\left(t_{1}, \cdots, t_{k-1}, 0, t_{k+1}, \cdots, t_{n}\right), \quad(t)_{k}=\left(t_{1}, \cdots, t_{k-1}, t_{k+1}, \cdots, t_{n}\right) \in$ $R^{n-1}$ and where $u(\cdot) \in C^{(\alpha)}\left(\bar{R}_{+}^{n} ; E\right)$, (the condition $u(t) \in D(A)$ is void since $D(A)=E)$. We shall assume that $\Phi=\left\{\eta_{\beta, k}\right\}$ is such that there exists a $u \in C^{(\alpha)}\left(\bar{R}_{+}^{n} ; E\right)$ satisfying (4.14). This can be assured by means of intrinsic conditions on the family $\Phi$ that reduce to differentiability requirements and to the compatibility condition (4.3) when $\alpha=(1,1)$. (For instance, when $\alpha=(1,1, \cdots, 1) \Phi$ consists of $n$ functions $\eta_{1}, \eta_{2}, \cdots, \eta_{n}$ defined in $\bar{R}_{+}^{n-1}$; then the assumption above reduces to $\eta_{k} \in C^{(1)}\left(\bar{R}_{+}^{n-1} ; E\right)$ for $1 \leqq k \leqq n$,

$$
\eta_{k}\left(\left\langle(t)_{k}\right\rangle_{j}\right)=\eta_{j}\left(\left\langle(t)_{j}\right\rangle_{k-1}\right)
$$

for $1 \leqq j<k \leqq n)$. Let $\hat{v}$ denote the extension of $v$ to all of $R^{n}$, $\left(\widehat{v}(t)=0\right.$ if $\left.t \notin \bar{R}_{+}^{n}\right)$, and let $\widehat{D^{\alpha} v}$ be $D^{\alpha} v$ similarly extended. Finally, let $T \in \mathscr{D}^{\prime}\left(R^{n} ; E\right)$ be the distribution-with support in the boundary of $\bar{R}_{+}^{n}$-defined by

$$
T=D^{\alpha} \widehat{v}-\widehat{D^{\alpha} v} .
$$

Then it is easy to see that $T$ depends only on the functions in $\Phi$ and their derivatives-and not on $v$. Define now

$$
u(t)=\left(S_{\alpha} * T\right)(t)
$$

$t \in \bar{R}_{+}^{n}$. A simple computation basically similar to the one for the case $\alpha=(1,1)$ shows that $u(\cdot)$ is a solution of $(4.13)-(4.14)$. Uniqueness and continuous dependence on initial conditions follow also in the same way; part (b) of Theorem 4.1 reads now

$\left(b^{\prime}\right)$ Let $\left\{u_{\nu}(\cdot)\right\}$ be a generalized sequence of strong solutions of (4.11) such that

$$
D^{\beta} u_{\nu}\left(\langle\cdot\rangle_{j}\right) \rightarrow 0
$$

uniformly on compacts of $\bar{R}_{+}^{n-1}$ for $\beta \leqq \alpha-e_{\jmath}, j=1,2, \cdots, n$. Then $u_{\nu}(\cdot) \rightarrow 0$ uniformly on compacts of $\overline{R_{+}^{n}}$.

The proof of $\left(b^{\prime}\right)$ is based, as it was the case for (b), on formula (4.16), which can directly be shown to hold for any solution of (4.13)(4.14).

Note, finally, that an analogous of Remark 4.2 holds as well for the case $|\alpha| \geqq 3$.

5. The case $|\alpha|=2$. The only possible choices for $\alpha$ are here $\alpha=(1,1)(n=2)$ or $\alpha=2(n=1)$. The main result in this isection is

TheOREM 5.1. $\Theta((1,1))=\Theta(2), \Xi((1,1)) \supseteqq \Xi(2)$.

The proof is a consequence of the following 
AUXILIARY LEMMA 5.2. Let $f(\cdot)$ be an F-valued function, defined and twice continuously differentiable in $[-a, a], a>0$. Define

$$
u\left(t_{1}, t_{2}\right)=\frac{2}{\pi} \int_{-1}^{1}\left(1-\eta^{2}\right)^{-1 / 2} f\left(2\left(t_{1} t_{2}\right)^{1 / 2} \eta\right) d \eta .
$$

Then $u$ is defined and has continuous partial derivatives $D_{1} u, D_{2} u$, $D_{1} D_{2} u$ in

$$
H(a)=\left\{\left(t_{1}, t_{2}\right) \in \bar{R}_{+}^{2} ; t_{1} t_{2}<a^{2} / 4\right\} ;
$$

in particular,

$$
D_{1} D_{2} u\left(t_{1}, t_{2}\right)=\frac{2}{\pi} \int_{-1}^{1}\left(1-\eta^{2}\right)^{-1 / 2} f^{\prime \prime}\left(2\left(t_{1} t_{2}\right)^{1 / 2} \eta\right) d \eta .
$$

Proof. Let $\left(t_{1}, t_{2}\right) \in H(a)$ and assume in addition that $t_{1} t_{2}>0$. Differentiating (5.1) under the integral sign-which is permissiblewe obtain

$$
\begin{aligned}
D_{1} u\left(t_{1}, t_{2}\right) & =\frac{2}{\pi}\left(\frac{t_{2}}{t_{1}}\right)^{1 / 2} \int_{-1}^{1}\left(1-\eta^{2}\right)^{-1 / 2} \eta f^{\prime}\left(2\left(t_{1} t_{2}\right)^{1 / 2} \eta\right) d \eta \\
& =\frac{4 t_{2}}{\pi} \int_{-1}^{1}\left(1-\eta^{2}\right)^{1 / 2} f^{\prime \prime}\left(2\left(t_{1} t_{2}\right)^{1 / 2} \eta\right) d \eta
\end{aligned}
$$

where we have used the fact that $\left[\left(1-\eta^{2}\right)^{1 / 2}\right]^{\prime}=-\left(1-\eta^{2}\right)^{-1 / 2} \eta$ and integrated by parts the first integral. Since $u$ is symmetric in $t_{1}, t_{2}$, $D_{2} u\left(t_{1}, t_{2}\right)$ can be obtained by interchanging these variables in (5.4). Differentiating now the first integral in (5.4) with respect to $t_{2}$, integrating by parts one of the integrals in the same way as before and using the equality $\left(1-\eta^{2}\right)^{-1 / 2}=\left(1-\eta^{2}\right)^{1 / 2}+\left(1-\eta^{2}\right)^{-1 / 2} \eta^{2}$ we obtain (5.3). It is clear from (5.3) and from the second integral in (5.4) that $D_{1} u, D_{2} u, D_{1} D_{2} u$ exist and are continuous even when $t_{1} t_{2}=0$, as claimed.

REMARK 5.3. It should be pointed out that Lemma 5.2 remains valid if the assumption of continuity of $f^{\prime \prime}$ is replaced by piecewise continuity. The proof is essentially the same. This will be used later in the proof of the inclusion $\Xi(2) \subseteq \Xi((1,1))$.

Proof of Theorem 5.1. It was proved in $\S 3$ (see (3.13)) that $\Theta(\alpha) \leqq \Theta(|\alpha|)$ for any $\alpha \in Z_{+}^{n}$; in particular

$$
\Theta((1,1)) \leqq \Theta(2) \text {. }
$$

To prove the reverse inclusion, let $A \in \Theta(2)$ and let

$$
S_{2} \in \mathscr{D}^{\prime}\left(R ; \mathscr{L}(E, D(A))_{f}\right.
$$


be a solution of

$$
S_{2}^{\prime \prime}-A S_{2}=\delta_{1} \otimes I
$$

commuting with $A$ and with support in $t \geqq 0$. Let now $f_{n}(\cdot)$, $n=1,2, \cdots$ be continuous $\mathscr{L}(E, D(A))$-valued functions defined (say) in $(-n, n)$ and such that

$$
S_{2}=f_{n}^{(p(n))}
$$

in $(-n, n)$ for some $p(n) \in Z_{+}, n=1,2, \cdots$ (the existence of such a sequence is assured by the fact that $S_{2}$ is of finite order locally). By integrating $f_{n}$-and thus raising $p(n)$-we may plainly suppose that $f_{n}$ is twice continuously differentiable in $(-n, n)$, that $p(1) \leqq$ $p(2) \leqq \cdots$ and that each $p(n)$ is odd. By Theorem 2.0 we may also assume that $f_{n}(t)=0$ for $t<0$ for all $n$.

Consider now the equation (5.5) in $(-n, n)$. Integrating both sides $p(n)$ times-that is, convoluting with $Y_{p(n)}$-we obtain

$$
f_{n}^{\prime \prime}(t)-A f_{n}(t)=\frac{(h(t) t)^{p(n)-1}}{(p(n)-1) !} I
$$

in the sense of distributions and, a fortiori, in the ordinary sense (all the functions involved in (5.7) are continuous). Let now $u_{n}\left(t_{1}, t_{2}\right)$, $n=1,2, \cdots$ be the function defined in $H(n)$ by (5.1) (with $f=f_{n}$ ) and extended to its complement by setting $u_{n}=0$ there. For $\varphi \in \mathscr{D}\left(R^{2}\right)$ define

$$
S(\varphi)=\left(D_{1}^{q(n)} D_{2}^{q(n)} u_{n}\right)(\varphi)
$$

where the derivatives are understood in the sense of distributions, $n$ is such that

$$
\operatorname{supp}(\varphi) \cap \bar{R}_{+}^{2} \subseteq H(n)
$$

and $q(n)=1 / 2(p(n)+1)$. To see that the prescription (5.8) is independent of $n$, let $m, n$ (say, $m>n$ ) both satisfy (5.6). Since

$$
S_{2}=f_{n}^{(p(n))}=f_{m}^{(p(m))}
$$

in $(-n, n)$,

$$
f_{m}^{(j)}=f_{n}
$$

where $j=p(m)-p(n)$ is an even number, $j=2 k$. Consequently, in view of the definition of $u_{n}, u_{m}$ and of Lemma 5.2 (applied repeatedly if necessary)

$$
D_{1}^{k} D_{2}^{k} u_{m}=u_{n}
$$


in $H(n) \cup \mathscr{C} R_{+}^{n}$ in the sense of distributions ( $\mathscr{C}$ indicates complement). Applying $D_{1}^{\gamma(n)} D_{2}^{\gamma(n)}$ to both sides of $(5.10)$ we obtain

$$
D_{1}^{q(n)} D_{2}^{q(n)} u_{n}=D_{1}^{q(m)} D_{2}^{q(m)} u_{m}
$$

in $H(n) \cup \mathscr{C} R_{+}^{n}$ as desired. It is evident from the definition of the topology of $\mathscr{D}\left(R^{2}\right)$ that $S$-as defined by (5.8)-belongs to $\mathscr{D}^{\prime}\left(R^{2} ; \mathscr{L}(E, D(A))\right.$; it is also plain that it has support in $\bar{R}_{+}^{2}$, commutes with $A$ and is locally of finite order. Finally, we go back to (5.7), to the definition of $u_{n}$ and to Lemma 5.2. According to them

$$
D_{1} D_{2} u_{n}-A u_{n}=K_{n}\left(h\left(t_{1}\right) t_{1}\right)^{q(n)-1}\left(h\left(t_{2}\right) t_{2}\right)^{q(n)-1} I
$$

in $H(n) \cup \mathscr{C} R_{+}^{n}$, where

$$
\begin{aligned}
K_{n} & =\frac{2^{p(n)}}{\pi(p(n)-1) !} \int_{0}^{1}\left(1-\eta^{2}\right)^{-1 / 2} \eta^{p(n)-1} d \eta \\
& =\frac{2^{p(n)-1}}{\pi(p(n)-1) !} B\left(\frac{1}{2}, \frac{1}{2} p(n)\right)=\frac{1}{((q(n)-1) !)^{2}}
\end{aligned}
$$

(see [10], formulas 4 and 5 in 8.384). Consequently, (5.11) can be written

$$
\left(D_{1} D_{2}-A\right) u_{n}=\left(Y_{q(n)} \otimes Y_{q(n)}\right) \otimes I .
$$

Differentiating $q(n)$ times with respect to each variable and using (5.8) we see that $S$ satisfies

$$
\left(D_{1} D_{2}-A\right) S=\left(\delta_{1} \otimes \delta_{1}\right) \otimes I
$$

in $H(n) \cup \mathscr{C} R_{+}^{2}$; as $n$ is arbitrary (5.12) holds in $R^{2}$, which shows that $A \in \Theta((1,1))$.

The proof that $\Xi(2) \subseteq \Xi((1,1))$ runs much along the same lines: in fact, let $S_{2}$ in (5.5) be a $\mathscr{L}(E, E)$-valued function such that $S_{2}(\cdot) u \in C^{(1)}\left(\bar{R}_{+} ; E\right)$ for all $u \in E$. Then one can choose $p(n)$ in (5.6) to be equal to 1 for all $n, f(t)=\int_{0}^{t} S_{2}(s) d s$ for $t \geqq 0, f(t)=0$ for $t<0$. Accordingly, formula (5.8) can be written

$$
S=D_{1} D_{2} \frac{2}{\pi} \int_{-1}^{1}\left(1-\eta^{2}\right)^{-1 / 2} \int_{0}^{2\left(t_{1} t_{2}\right)^{1 / 2} \eta} S_{2}(\xi) d \xi
$$

Applying the preceding formula to an $u \in E$, taking advantage of the fact that $S_{2}(\cdot) u \in C^{(1)}$ and of Lemma 5.2 (see also Remark 5.3) we obtain

$$
S\left(t_{1}, t_{2}\right) u=\frac{2}{\pi} \int_{-1}^{1}\left(1-\eta^{2}\right)^{-1 / 2} S_{2}^{\prime}\left(2\left(t_{1} t_{2}\right)^{1 / 2} \eta\right) u d \eta
$$

for any $t_{1}, t_{2} \geqq 0$; therefore $S$ is strongly continuous there and 
$A \in \Xi((1,1))$. This ends the proof of Theorem 5.1 .

REMARK 5.4. It is not in general true-except for obvious exceptional cases like $\operatorname{dim} E<\infty$ - that $\Xi((1,1))=\Xi(2)$. To examine this question we study in detail the case where $E=H$ is a Hilbert space and $A$ is a normal, possibly unbounded operator in $H$. We shall make some use in what follows of the functional calculus for these operators; although only the self adjoint case is examined in detail in [12] the results to be used can be extended easily to normal operators.

For any $\eta \geqq 0$ we define

$$
\kappa(\eta)=\kappa(\eta ; A)=\text { ess. } \sup \left\{\left|I_{0}(\eta \lambda)\right| ; \lambda^{2} \in \sigma(A)\right\}
$$

where the essential supremum is taken with respect to the spectral resolution $\{P(\cdot)\}$ of $A$ and $I_{0}$ is the Bessel function

$$
I_{0}(\lambda)=\sum_{n=0}^{\infty} \frac{1}{(n !)^{2}}\left(\frac{\lambda}{2}\right)^{2 n}
$$

([10], formula 8.447.1); since no assumption is made for the time being on $\sigma(A)$, we admit the value $+\infty$ for $\kappa$. Some information about how $\sigma(A)$ must be to insure finiteness of $\kappa$ can be obtained from asymptotic developments for $I_{0}(\lambda)$. In fact, it follows from [10], formula 8.451.1 that, as $|\lambda| \rightarrow \infty$

$$
I_{0}(\lambda)=(2 \pi \lambda)^{-1 / 2}\left(e^{\lambda} \pm i e^{-\lambda}\right)(1+o(1))
$$

where the $+(-)$ sign is to be used in the upper (lower) half-plane and the branch of the square root is the one discontinuous in the negative real axis. According to (5.14), there exist positive constants $K_{1}, K_{2}, K_{3}$ such that

$$
K_{1}\left|I_{0}(\eta \lambda)\right| \leqq \eta^{-1 / 2}|\lambda|^{-1 / 2} e^{\eta|R e \lambda|} \leqq K_{2}\left|I_{0}(\eta \lambda)\right|+K_{3}
$$

for, say, $|\eta \lambda| \geqq 1$. Then, if we define

$$
\tilde{\kappa}(\eta)=\operatorname{ess} \cdot \sup \left\{|\lambda|^{-1 / 2} e^{\eta|R e \lambda|} ; \lambda^{2} \in \sigma(A),|\lambda| \geqq 1\right\}
$$

for $\eta \geqq 0$ we have

$$
K_{1} \kappa(\eta) \leqq \eta^{-1 / 2} \tilde{\kappa}(\eta) \leqq K_{2} \kappa(\eta)+K_{3}
$$

for $\eta \geqq 1$. Since, on the other hand,

$$
\tilde{\kappa}(\eta) \leqq \tilde{\kappa}\left(\eta^{\prime}\right)
$$

for $\eta \leqq \eta^{\prime}$ we see that $\kappa(\cdot)$ is bounded on compacts of $\eta \geqq 0$ if and only if $\tilde{\kappa}(\eta)<+\infty$ for all $\eta \geqq 0$. 
TheOREM 5.5. Let $H, A$ be as above. Then $A \in \Xi((1,1))$ if and only if

$$
\tilde{\kappa}(\eta)<+\infty
$$

for all $\eta \geqq 0$.

Proof. Assume (5.15) holds. For $t_{1}, t_{2} \geqq 0$ let

$$
f\left(t_{1}, t_{2}, \lambda\right)=I_{0}\left(2\left(t_{1} t_{2} \lambda\right)^{1 / 2}\right)
$$

where, by virtue of the definition (5.13) of $I_{0}$ the choice of branch of the square root is immaterial. Define

$$
S\left(t_{1}, t_{2}\right)=f\left(t_{1}, t_{2}, A\right)=\int_{\sigma(A)} f\left(t_{1}, t_{2}, \lambda\right) P(d \lambda)
$$

By virtue of (5.15) and [12], Chapter IX, $\S 128 S\left(t_{1}, t_{2}\right)$ is a bounded operator in $H$; more precisely,

$$
\left|S\left(t_{1}, t_{2}\right)\right|=\text { ess. } \sup \left\{\left|f\left(t_{1}, t_{2} ; \lambda\right)\right| ; \lambda \in \sigma(A)\right\}=\kappa\left(2\left(t_{1} t_{2}\right)^{1 / 2}\right) \text {. }
$$

The second equality in (5.17), the facts that $\kappa$ is bounded on compacts of $\eta \geqq 0$ and that $f$ is continuous in $\bar{R}_{+}^{2}$ for $\lambda$ fixed yield, by a simple application of the Lebesgue dominated convergence theorem that $S(\cdot)$ is strongly continuous in $\bar{R}_{+}^{2}$.

We apply now the results of $\S 3$ (in $E=R$ ) to the function $f$. According to them,

$$
\left(D_{1} D_{2}-\lambda\right) f(\cdot, \lambda)=\delta
$$

where $\delta$ is the Dirac measure in $R^{2}$ (here we have set $f=0$ outside of $\left.\bar{R}_{+}^{2}\right)$. But then we obtain by applying (5.18) to an arbitrary test function $\varphi \in \mathscr{D}\left(R^{n}\right)$, integrating the result with respect to $P(\cdot)$ and making use of Fubini's theorem that $S$-extended to the outside of $\bar{R}_{+}^{2}$ by setting $S=0$ there-satisfies

$$
\left(D_{1} D_{2}-A\right) S=\delta \otimes I
$$

We have thus shown that $A \in \Xi((1,1)$ ) (the fact $S$ commutes with $A$ is trivial). Conversely, assume $A \in \Xi((1,1))$ and let $\hat{S}$ be the strongly continuous, $\mathscr{L}(E, E)$-valued function with support in $\bar{R}_{+}^{2}$, commuting with $A$ and satisfying (5.19). We can still define $S\left(t_{1}, t_{2}\right)$ for $t_{1}, t_{2} \geqq 0$ by means of (5.16); this time, $S\left(t_{1}, t_{2}\right)$ will only be a closed, densely defined operator ([12], Chapter IX, § 127); for every $t_{1}, t_{2} \geqq 0 D\left(S\left(t_{1}, t_{2}\right)\right.$ ) contains the subspace $F$ generated by all elements of the form 
where $e$ is any bounded Borel set in the complex plane, $u$ any element of $H$. Now, since for $u \in F$ the domain of integration in (5.16) is bounded, it can be proved with arguments similar to the ones used above that if $u \in F$ then $S(\cdot) u \in C^{(1,1))}\left(\bar{R}_{+}^{2} ; H\right)$ and satisfies

$$
\left(D_{1} D_{2}-A\right) S u=\delta \otimes u .
$$

By uniqueness of solutions of the Goursat problem (Theorem 2.1) we must have

$$
S u=\hat{S} u
$$

as distributions, and a fortiori pointwise in $\bar{R}_{+}^{2}$ for any $u \in F$. But $F$ is dense in $H$, thus (5.20) implies that $S\left(t_{1}, t_{2}\right)$ is bounded for all $\left(t_{1}, t_{2}\right) \in \bar{R}_{+}^{2}$. Applying the results in [12], Chapter IX, $\S 128$ combined with the second equality in (5.17) we see that $\kappa(\eta)$-thus $\tilde{\kappa}(\eta)$-is finite for all $\eta \geqq 0$ which ends the proof of Theorem 5.5.

To give now an example of an operator that belongs to $\Xi((1,1))$ but not to $\Xi(2)$ is simple. In fact, let $A$ be, say, any normal operator whose spectrum $\sigma(A)$ consists of the region

$$
\Omega=\left\{\lambda ;\left|R e \lambda^{1 / 2}\right| \leqq \max \left(0, \log \log |\lambda|{ }^{1 / 2}\right)\right\}
$$

(we may take, for instance, $H=L^{2}(\Omega)$ with respect to ordinary Lebesgue measure in the plane, $A$ the operator of "multiplication by the variable"). Then, if $\eta \geqq 0$,

$$
\tilde{\kappa}(\eta) \leqq \sup \left\{(\log |\lambda|)^{\eta}|\lambda|^{-1 / 2} ;|\lambda| \geqq 1\right\}<\infty
$$

which shows that $A \in \Xi((1,1))$. Assume now $A \in \Xi(2)$. According to [4], Remark 5.7 and Theorem $5.9 \sigma(A)$ must be contained in a region of the form

$$
\left\{\lambda ;\left|\operatorname{Re} \lambda^{1 / 2}\right| \leqq \omega\right\}
$$

for some $\omega<\infty$. But $\Omega$ cannot be described in this way, thus $A \notin \Xi(2)$.

It might be pointed out that in some cases of interest the implication $A \in \Xi((1,1)) \Rightarrow A \in \Xi(2)$ holds. For instance, assume that $A$ is an everywhere defined and continuous member of $\Theta((1,1))$, (see $\S 1$ for comments on this) and let $S \in \mathscr{D}^{\prime}\left(R^{2}, \mathscr{L}(E, E)\right)_{f}$ be the solution of (5.19) with support in $\bar{R}_{-}^{2}$. Finally, let $f$ be a continuous, $\mathscr{L}(E, E)$-valued function defined in, say, $\left|t_{k}\right|\langle a, a\rangle 0$, and satisfying there

$$
S=D_{1}^{p_{1}} D_{2}^{p_{2}} f
$$

for some $p=\left(p_{1}, p_{2}\right) \in \bar{Z}_{+}^{2}$. By Theorem 2.0 we may assume that 
$f=0$ in $\mathscr{C} \bar{R}_{+}^{2}$, the complement of $\bar{R}_{+}^{2}$. Reasoning as in the comments preceding and following (5.7) we see that we may assume $f$ to belong to $C^{((1,1))}$ in $\left|t_{k}\right|<a$ and that we must then have

$$
D_{1} D_{2} f(t)=A f(t)+\frac{\left(h\left(t_{1}\right) t_{1}\right)^{p_{1}-1}\left(h\left(t_{2}\right) t_{2}\right)^{p_{2}-1}}{\left(p_{1}-1\right) !\left(p_{2}-1\right) !} I
$$

in $\left|t_{k}\right|<a$. But since $A \in \mathscr{L}(E, E),(5.22)$ implies that $D_{1} D_{2} f \in C^{((1,1))}$ in the intersection of $\bar{R}_{+}^{2}$ and $\left|t_{k}\right|<a$; reasoning in this way repeatedly we see that $f$ has continuous partials of any order there, and thus the same is true of $S$. Since one can argue likewise for any $a>0$, $S$ has continuous partials of any order in all of $\bar{R}_{+}^{2}$ (in particular, $A$ belongs to $\Xi((1,1)))$. We now go back to $\S 3$; according to the results there, if $\widetilde{S}$ is the distribution defined from $S$ by the formula (3.11) then $\widetilde{S} \in \mathscr{D}^{\prime}(R ; \mathscr{L}(E, E))$, has its support in $t \geqq 0$ and satisfies

$$
\widetilde{S}^{\prime \prime}-A S=\delta_{1} \otimes I \text {. }
$$

But, according to the comments following (3.11) if $S$ is a function, $\widetilde{S}$ is given by the formula (3.12) which in our case takes the form

$$
\widetilde{S}(t)=\int_{0}^{t} S(t-s, s) d s, \quad \mathrm{t} \geqq 0 .
$$

This clearly shows that $A \in \Xi(2)$.

6. The Banach space case. The method used here is merely an adaptation of the one used by Chazarain in [3] for the case of one variable. Recall that a logarithmic region $\Lambda=\Lambda(\sigma, \tau, \omega)$ in the complex plane $C$ is any subset of $C$ defined by an inequality of the type

$$
\operatorname{Re} \lambda \geqq \max (\sigma \log |\operatorname{Im} \lambda|+\tau, \omega)
$$

where $\sigma, \tau, \omega$ are real constants, $\sigma>0$.

THEOREM 6.1. Let $\alpha \in Z_{+}^{n}$, and assume that $E$ is a Banach space. Then $A \in \Theta(\alpha)$ if and only if $R\left(\lambda^{|\alpha|} ; A\right)=\left(\lambda^{|\alpha|} I-A\right)^{-1}$ exists for $\lambda$ in some logarithmic region $A$ and

$$
\left|R\left(\lambda^{|\alpha|} ; A\right)\right| \leqq p(|\lambda|)
$$

there, where $p(\cdot)$ is a polynomial.

Before proceeding to the proof a few comments may be useful. Observe first that if $m>2$ the set $\left\{\lambda^{m} ; \lambda \in \Lambda\right\}, \Lambda$ any logarithmic region contains a neighborhood of $\infty$, thus the conclusion of Theorem 6.1 simply means that $A$ is bounded-which is, of course, in accordance 
with Theorem 3.2. Second, the condition that $S_{\alpha}$ should be of finite order locally in the definition of the class $\Theta(\alpha)$ is now void; in fact, since $E$-thus $D(A)$-is a Banach space, so is $\mathscr{L}(E, D(A))$; then, according to [13], Corollaire 2, p. 85 every distribution with values in $\mathscr{L}(E, D(A))$ is of finite order locally. Finally, Theorem 6.1 could be fairly easily read out from the results in the preceding section and those in [3]; however, to deduce them anew seems justified not only by the importance of the particular case considered but also because substantial simplifications become available.

Proof of Theorem 6.1. Assume $A \in \Theta(\alpha)$; let $S_{\alpha} \in$ $\mathscr{D}^{\prime}\left(R^{n} ; \mathscr{L}(E, D(A))\right.$ be the solution of

$$
\left(D^{\alpha}-A\right) S_{\alpha}=\delta \otimes I
$$

commuting with $A$ and with support in $\bar{R}_{+}^{n}$. Given $a \in R_{+}^{n}$ let $f$ be a continuous function in $\left|t_{k}\right|<a_{k}, p \in Z_{+}^{n}$ such that

$$
S_{\alpha}=D^{p} f
$$

in $\left|t_{k}\right|<a_{k}$. Applying Theorem 2.0 we may suppose that $f=0$ in $\mathscr{C} \bar{R}_{+}^{n}$. Let now $\varphi \in \mathscr{D}\left(R^{n}\right)$ with support in $\left|t_{k}\right|<a_{k}$ and such that $\varphi(t) \equiv 1$ in $\left|t_{k}\right| \leqq b_{k}, 0<b_{k}<a_{k}, 1 \leqq k \leqq n$. Define

$$
\varphi_{\lambda}(t)=e^{-(\lambda, t)} \varphi(t)
$$

where $\lambda=\left(\lambda_{1}, \cdots, \lambda_{n}\right)$ is an element of $C^{n}$, $n$-dimensional unitary space and $(\lambda, t)=\lambda_{1} t_{1}+\cdots+\lambda_{n} t_{n}$. Plainly $\varphi_{i} \in \mathscr{D}\left(R^{n}\right)$ for all $\lambda \in C^{n}$. Now define

$$
R(\lambda)=S\left(\varphi_{\lambda}\right)
$$

Then $R(\lambda) \in \mathscr{L}(E, D(A))$ and commutes with $A$. Observe next that we have

$$
D^{\alpha} \varphi_{\lambda}(t)=\sum K_{\hat{\beta}}\left(D^{3} e^{-(\lambda, t)}\right)\left(D^{r} \varphi\right)(t)=\sum K_{\hat{\beta}}(-\lambda)^{\beta} e^{-(\lambda, t)}\left(D^{\prime} \varphi\right)(t)
$$

where the summation is extended over all $\beta, \gamma \in \bar{Z}_{+}^{n}$ such that $\beta+\gamma=$ $\alpha$, and $K_{\beta}=\left(\begin{array}{l}\alpha_{1} \\ \beta_{1}\end{array}\right) \cdots\left(\begin{array}{l}\alpha_{n} \\ \beta_{n}\end{array}\right)$ (in the second sum we have used the notation $\mu^{3}$ for the product $\mu_{1}^{\beta_{1}} \cdots \mu_{n}^{\beta_{n}}, \mu=\left(\mu_{1}, \cdots, \mu_{n}\right) \in C^{n}$. Consequently (and on the basis of (6.2)) $R(\lambda)$ satisfies

$$
\left(\lambda^{\alpha} I-A\right) R(\lambda)=I-M(\lambda)
$$

where now

$$
M(\lambda)=S\left(\psi_{\lambda}\right), \quad \psi_{\lambda}(t)=D^{\alpha} \varphi_{\lambda}(t)-(-\lambda)^{\alpha} \mathcal{P}(t) .
$$

Observe now that, by virtue of (6.3) we can write (after some 
integrations by parts)

$$
M(\lambda)=\int\left[(-1)^{|p|} D^{p} \psi_{\lambda}(t)\right] f(t) d t .
$$

The partial $D^{p} \psi_{\lambda}$ can be explicitly computed by using an expression of the type of (6.5); the result will be a sum each of whose terms will have the form

$$
K \lambda^{\beta} e^{-(\lambda, t)} D^{r} \varphi(t)
$$

where $\beta, \gamma \in \bar{Z}_{+}^{n}, \beta+\gamma=\alpha+p$. Note that in the expression (6.7) for $\psi_{\lambda}$-developed by means of (6.5)-we always have $\gamma \neq 0$; therefore the same is true of each of the terms (6.8), which means that $D^{p} \psi_{\lambda}(t)=0$ for $\left|t_{k}\right|<b_{k}$. Accordingly, if $\left|R e \lambda_{k}\right| \geqq \varepsilon>0$

$$
|M(\lambda)| \leqq K^{\prime} \int\left|D^{p} \psi_{\lambda}(t)\right| d t \leqq K^{\prime \prime} \prod_{k=1}^{n}\left|\lambda_{k}\right|^{\alpha_{k}+p_{k}} e^{-b_{k} B e \lambda_{i k}}
$$

for appropriate constants $K^{\prime}, K^{\prime \prime}$. Now let $\rho$ be any positive number less than 1. Some simple manipulations show that there exists a logarithmic region $A$ such that if $\lambda_{k} \in \Lambda, 1 \leqq k \leqq n$, then the righthand side of (6.9) does not surpass $\rho$. Accordingly, $|M(\lambda)| \leqq \rho$ for $\lambda_{k} \in \Lambda$. But this implies that $I-M(\lambda)$ has a bounded inverse $(I-M(\lambda))^{-1}=\sum_{j=0}^{\infty} M(\lambda)^{j}$ there. Since $M(\lambda)$ commutes with $A$ (immediate consequence of the corresponding property for $S_{\alpha}$ and of the definition of $M(\lambda)$, so does $(I-M(\lambda))^{-1}$. Post multiplying (6.6) by it we obtain

$$
\left(\lambda^{\alpha} I-A\right) R(\lambda)(I-M(\lambda))^{-1}=I
$$

which, together with the commutativity property just mentioned implies $R(\lambda)(1-M(\lambda))^{-1}=R\left(\lambda^{\alpha} ; A\right)$ for $\lambda_{k} \in A$. Observe now that

$$
\left|(I-M(\lambda))^{-1}\right| \leqq(1-\rho)^{-1}
$$

for $\lambda_{k} \in \Lambda$. As for $R(\lambda)$, a bound for its norm can be obtained much in the same way as (6.9) was obtained. In fact, combining (6.3) and (6.4) we can write

$$
R(\lambda)=\int\left[(-1)^{|x|} D^{p} \varphi_{\lambda}(t)\right] f(t) d t .
$$

Developing now $D^{p} \varphi_{i}$ according to (6.5) we obtain

$$
|R(\lambda)| \leqq K \prod_{k=1}^{n}\left|\lambda_{k}\right|^{p_{k}}
$$

for a suitable constant $K$. Combining this with (6.10) we see that for $\lambda_{k} \in \Lambda, \lambda=\left(\lambda_{1}, \cdots, \lambda_{n}\right), R\left(\lambda^{\alpha} ; A\right)$ exists and 


$$
\left|R\left(\lambda^{\alpha} ; A\right)\right| \leqq K(1-\rho)^{-1} \prod_{k=1}\left|\lambda_{k}\right|^{p_{k}}
$$

If we now take $\lambda_{1}=\cdots=\lambda_{n}=\lambda \in \Lambda$, the conclusion of Theorem 6.1 follows.

Assume now that $A$ is such that $R\left(\lambda^{|\alpha|} ; A\right)$ exists for $\lambda$ in a logarithmic region $\Lambda$ and satisfies (6.1) there. If $|\alpha|>2$ it was already observed that $A$ must belong to $\mathscr{L}(E, E)=\Phi(\alpha)$, thus Theorem 3.2 applies to show that $A \in \Theta(\alpha)$. Let then $|\alpha| \leqq 2$; there are only three such cases, namely $\alpha=1, \alpha=2, \alpha=(1,1)$. Since the first two cases correspond to the Cauchy problem (and are, besides, treated in detail in [3]) we only consider the last. It was proved in [3] that if $R\left(\lambda^{2} ; A\right)$ exists for $\lambda$ in a logarithmic region $A$ and (6.1) holds, then $A \in \Theta(2)$. According then to the results in $\S 5$-in particular, Theorem 5.1-A belongs as well to $\Theta((1,1))$. This ends the proof of Theorem 2.1.

REMARK 6.2. It is possible to give a fairly explicit expression for $S_{\alpha}(\varphi), \varphi \in \mathscr{D}\left(R^{n}\right)$ in the present case. We do it in detail only for $\alpha=(1,1)$, the extension to other values of $\alpha$ being simple.

Assume $A \in \Theta((1,1))$. It was proved during the course of the demonstration of Theorem 6.1 that there exists a logarithmic region $\Lambda=\Lambda(\sigma, \tau, \omega)$ such that, if $\lambda_{1}, \lambda_{2} \in \Lambda$ then $R\left(\lambda_{1} \lambda_{2} ; A\right)$ exists and satisfies there

$$
\left|R\left(\lambda_{1} \lambda_{2} ; A\right)\right| \leqq p\left(\left|\lambda_{1}\right|,\left|\lambda_{2}\right|\right)
$$

$p(\cdot, \cdot)$ a polynomial in two variables. Let $\varphi \in \mathscr{D}(R)$ and denote by $\hat{\phi}(\lambda), \lambda \in C^{2}$ its Laplace transform

$$
\hat{\varphi}(\lambda)=\int_{R^{2}} e^{(\lambda, t)} \varphi(t) d t
$$

Integrating by parts repeatedly we obtain, for any $\beta=\left(\beta_{1}, \beta_{2}\right) \in \bar{Z}_{+}^{2}$,

$$
\hat{\varphi}(\lambda)=\frac{(-1)^{|\beta|}}{\lambda^{\beta}} \int e^{(\lambda, t)} D^{\beta} \varphi(t) d t .
$$

Accordingly there exists for any such $\beta$ a constant $K=K(\beta)$ such that

$$
|\hat{\varphi}(\lambda)| \leqq K \prod_{k=1}^{2}\left(1+\left|\lambda_{k}\right|\right)^{-\beta_{k}} e^{a_{k} R e \lambda_{k}}
$$

where $a=\left(a_{1} a_{2}\right)$ is such that $\operatorname{supp}(\varphi) \cong-\bar{R}_{+}^{2}(-a)$. Let now $\Gamma_{k}$ be (say) the contours $R e \lambda_{k}=\max \left(\sigma \log \left|\operatorname{Im} \lambda_{k}\right|+\tau^{\prime}, \omega^{\prime}\right), k=1,2$ with $\tau^{\prime}>\tau, \omega^{\prime}>\omega$ and define 


$$
S(\varphi)=\int R\left(\lambda_{1} \lambda_{2} ; A\right) \hat{\varphi}(\lambda) d \lambda_{1} d \lambda_{2}
$$

the integral over $\Gamma_{1} \times \Gamma_{2}, \Gamma_{k}$ oriented clockwise with respect to $A$. With the help of (6.11) and (6.12)-for convenient $\beta$-it is easy to see that the prescription (6.13) defines a distribution in $\mathscr{D}^{\prime}\left(R^{n} ; \mathscr{L}(E, E)\right)$ commuting with $A$. Moreover, if $\operatorname{supp}(\varphi) \subset \mathscr{C} \bar{R}_{+}^{2}$, we can write $\varphi=\varphi_{1}+\varphi_{2}$ where $\varphi_{1}$ has support in $t_{1}<0, \varphi_{2}$ in $t_{2}<0$. This means that we can choose $a_{1}<0$ in the estimates (6.12) for $\varphi_{1}$ and then, "sliding towards $+\infty$ " the contour $\Gamma_{1}$ in (6.13), $S\left(\varphi_{1}\right)=0$. In the same way, $S\left(\varphi_{2}\right)=0$, thus $S(\varphi)=0$.

Accordingly, supp $(S) \subseteq \bar{R}_{+}^{2}$. Finally, it follows from the formulas

$$
\widehat{D_{1} D_{2} \varphi}(\lambda)=\lambda_{1} \lambda_{2} \hat{\varphi}(\lambda), \quad \lambda_{1} \lambda_{2} R\left(\lambda_{1} \lambda_{2} ; A\right)=I+A R\left(\lambda_{1} \lambda_{2} ; A\right)
$$

and from some elementary manipulations with (6.12) that $S$ belongs in fact to $\mathscr{D}^{\prime}\left(R^{2} ; \mathscr{L}(E, D(A))\right.$ and that it is a (and then the unique) solution of

$$
\left(D_{1} D_{2}-A\right) S=\delta \otimes I .
$$

The author is indebted to the referee for several improvements in the presentation of this paper.

\section{REFERENCES}

1. N. Bourbaki, Eléments de mathématique, fasc. XV, Espaces Vectoriels Topologiques, ch. 1-2, 2nd ed., Hermann, Paris, 1966

2. - Éléments de mathématique, fasc. XVIII, Espaces Vectoriels Topologiques, ch. 3-5, Hermann, Paris, 1955.

3. J. Chazarain, Problèmes de Cauchy au sens des distributions vactorielles et applications, C. R. Acad. Sci., Paris 266 (1968), 10-13.

4. H. O. Fattorini, Ordinary differential equations in linear topological spaces, I, J. Differential Equations, 5 (1969), 72-105.

5. - Ordinary differential equations in linear topological spaces II, J. Differential Equations 5 (1969), 50-70.

6. - On a class of differential equations for vector-valued distributions, Pacific J. Math., 32 (1970), 79-104.

7. A. Friedman, The Cauchy problem in several time variables, J. Math. Mech., 11 (1962), 859-889.

8. - Generalized functions and partial differential equations, Prentice-Hall, Inc., Englewood Cliffs, N. J., 1963.

9. I. M. Gel'fand and G. E. Shilov, Generalized functions and operations with them (Russian) Gostekhizdat, Moscow, 1958.

10. I. S. Gradstein and I. M. Ryzhik, Tables of integrals, sums, series and derivatives (Russian), 4th edition, Gostekhizdat, Moscow, 1963.

11. J. L. Lions, Les semigroupes distributions, Portugalia Math., 19 (1960), 141-164.

12. F. Riesz and B. Sz. and Nagy, Leçons d'analyse fonctionnelle, 3ème édition, Akadémiai Kiadó, Budapest, 1955.

13. L. Schwartz, Théorie des distributions, nouvelle édition, Hermann, Paris, 1966. 
14. - Théorie des distributions à valeurs vectorielles (I), Annales Inst. Fourier VII (1957), 1-141.

15. - Théorie des distributions à valeurs vectorielles (II), Annales Inst. Fourier VIII (1958), 1-209.

Received January 20, 1970. The preparation of this paper was supported in part by NSF Grant No. GP-9658.

University of CALifornia, Los ANgeles 



\section{PACIFIC JOURNAL OF MATHEMATICS}

\section{EDITORS}

H. SAMELSON

Stanford University

Stanford, California 94305

C. R. Новву

University of Washington

Seattle, Washington 98105
J. DUGundJI

Department of Mathematics

University of Southern California

Los Angeles, California 90007

RICHARD ARENS

University of California

Los Angeles, California 90024

\section{ASSOCIATE EDITORS}
E. F. BECKENBACH
B. H. NeumanN
F. WOLF
K. YOSHIDA

\section{SUPPORTING INSTITUTIONS}
UNIVERSITY OF BRITISH COLUMBIA
CALIFORNIA INSTITUTE OF TECHNOLOGY
UNIVERSITY OF CALIFORNIA
MONTANA STATE UNIVERSITY
UNIVERSITY OF NEVADA
NEW MEXICO STATE UNIVERSITY
OREGON STATE UNIVERSITY
UNIVERSITY OF OREGON
OSAKA UNIVERSITY
UNIVERSITY OF SOUTHERN CALIFORNIA
STANFORD UNIVERSITY
UNIVERSITY OF TOKYO
UNIVERSITY OF UTAH
WASHINGTON STATE UNIVERSITY
UNIVERSITY OF WASHINGTON
AMERICAN MATHEMATICAL SOCIETY CHEVRON RESEARCH CORPORATION TRW SYSTEMS
NAVAL WEAPONS CENTER 


\section{Pacific Journal of Mathematics}

\section{Vol. 37, No. $1 \quad$ January, 1971}

Gregory Frank Bachelis and Haskell Paul Rosenthal, On unconditionally

converging series and biorthogonal systems in a Banach space .........

Richard William Beals, On spectral theory and scattering for elliptic

operators with singular potentials .........................

J. Lennart (John) Berggren, Solvable and supersolvable groups in which every element is conjugate to its inverse ........................ 21

Lindsay Nathan Childs, On covering spaces and Galois extensions ..........

William Jay Davis, David William Dean and Ivan Singer, Multipliers and

unconditional convergence of biorthogonal expansions..............

Leroy John Derr, Triangular matrices with the isoclinal property ............

Paul Erdős, Robert James McEliece and Herbert Taylor, Ramsey bounds for

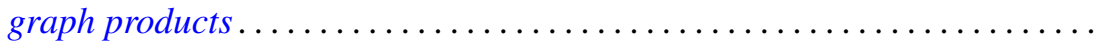

Edward Graham Evans, Jr., On epimorphisms to finitely generated

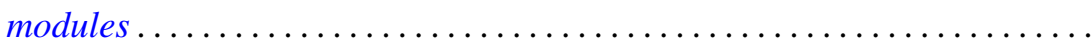

Hector O. Fattorini, The abstract Goursat problem ................. 51

Robert Dutton Fray and David Paul Roselle, Weighted lattice paths .........

Thomas L. Goulding and Augusto H. Ortiz, Structure of semiprime $(p, q)$

radicals ...........................................

E. W. Johnson and J. P. Lediaev, Structure of Noether lattices with join-principal maximal elements ....

David Samuel Kinderlehrer, The regularity of minimal surfaces defined over

slit domains

Alistair H. Lachlan, The transcendental rank of a theory. .

Frank David Lesley, Differentiability of minimal surfaces at the boundary ...

Wolfgang Liebert, Characterization of the endomorphism rings of divisible torsion modules and reduced complete torsion-free modules over complete discrete valuation rings....

Lawrence Carlton Moore, Strictly increasing Riesz norms.

Raymond Moos Redheffer, An inequality for the Hilbert transform ...

James Ted Rogers Jr., Mapping solenoids onto strongly self-entwined,

circle-like continua..........................

Sherman K. Stein, B-sets and planar maps ................... 217

Darrell R. Turnidge, Torsion theories and rings of quotients of Morita

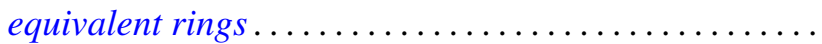

Fred Ustina, The Hausdorff means of double Fourier series and the principle of localization ................................

Stanley Joseph Wertheimer, Quasi-compactness and decompositions for arbitrary relations.

Howard Henry Wicke and John Mays Worrell Jr., On the open continuous images of paracompact $\check{C}$ ech complete spaces... 